\title{
Ru-catalyzed C-H functionalization of phenylglycine derivatives: synthesis of isoquinoline-1-carboxylates and isoindoline-1-carboxylates
}

Sara Ruiz, Francisco J. Sayago, Carlos Cativiela, Esteban P. Urriolabeitia*

Instituto de Síntesis Química y Catálisis Homogénea (ISQCH), CSIC-Universidad de Zaragoza, Facultad de Ciencias, Pedro Cerbuna 12, 50009 Zaragoza (Spain)

Corresponding author: Esteban P. Urriolabeitia (esteban@unizar.es). Postal address: ISQCH, Facultad de Ciencias, Edificio D, planta 4, Pedro Cerbuna 12, 50009 Zaragoza (Spain)

\begin{abstract}
The reaction of $N$-unprotected methylesters of phenylglycine derivatives (1a-1f) with electron-rich internal alkynes (2a-2e), catalyzed by $\left[\mathrm{Ru}(\text { cymene }) \mathrm{Cl}_{2}\right]_{2}(10 \%)$, gives the corresponding 3,4disubstituted isoquinoline-1-carboxylates 3 through $\mathrm{C}-\mathrm{H} / \mathrm{N}-\mathrm{H}$ oxidative coupling. The $\mathrm{C}-\mathrm{H}$ bond activation step is assisted by carboxylates, and $\mathrm{N}$-fluoro-2,4,6-trimethylpyridinium triflate works as the terminal oxidant. The process shows a remarkable tolerance to the presence of diverse electronreleasing and electron-attracting functional groups at the phenyl ring of the amino acid. In addition, the reaction of phenylglycine derivatives (1a-1f) with methyl acrylate (4a) catalyzed by $\left[\mathrm{Ru}(\text { cymene })_{2} \mathrm{Cl}_{2}\right]_{2}(10 \%)$ under the same experimental conditions, gives the corresponding 3, $N$ disubstituted isoindoline-1-carboxylates 5 through C-H/N-H coupling. Isoindolines 5 are obtained as a mixture of diastereoisomers, with moderate to high values of diastereomeric excess (up to $80 \%$ ).
\end{abstract}

\section{Keywords}

Ru-complexes / Amino acids / C-H activation / Isoquinolines / Isoindolines

\section{Introduction}

Amino acids are a group of molecules with an essential role in the development of life, because they are the basic structural components of peptides and proteins. The bonding pattern and the relative arrangement of amino acids change the properties of the peptide or the protein, up to the point of determining the way in which they are expressed. This close structure-activity relationship results in that controlled modifications of amino acids could be devised to promote tailored changes. Therefore the selective synthesis and modification of amino acids is a topic of high interest [1]. 
C-H functionalizations promoted by metal complexes have emerged as versatile, selective and often catalytic alternatives to classical and widely employed methods [2], providing in some cases alternative solutions [3]. Up to now, $\mathrm{C}\left(\mathrm{sp}^{2}\right)$-H activation of amino acids has focused on phenylalanine, as summarized in Figure 1 and, to a much lower extent, tryptophane and heterocyclic derivatives. Thus, Pd-catalyzed halogenations [4a], acetoxylations [4d], aminations [4a, 4b,4d,4f,4h], alkenylations $[4 \mathrm{a}, 4 \mathrm{i}]$ and carbonylations $[4 \mathrm{e}, 4 \mathrm{~g}]$ have been reported. In addition, one example of an Ir-catalyzed borylation, followed by Pd-mediated Suzuki coupling to yield arylated derivatives, has been reported [4c]. Very recently the catalytic functionalization of phenylglycine has been reported by $\mathrm{Yu}$ and coworkers (Figure 1), using Pd catalysts, but only a limited number of examples was studied $[4 \mathrm{j}, 4 \mathrm{k}]$.
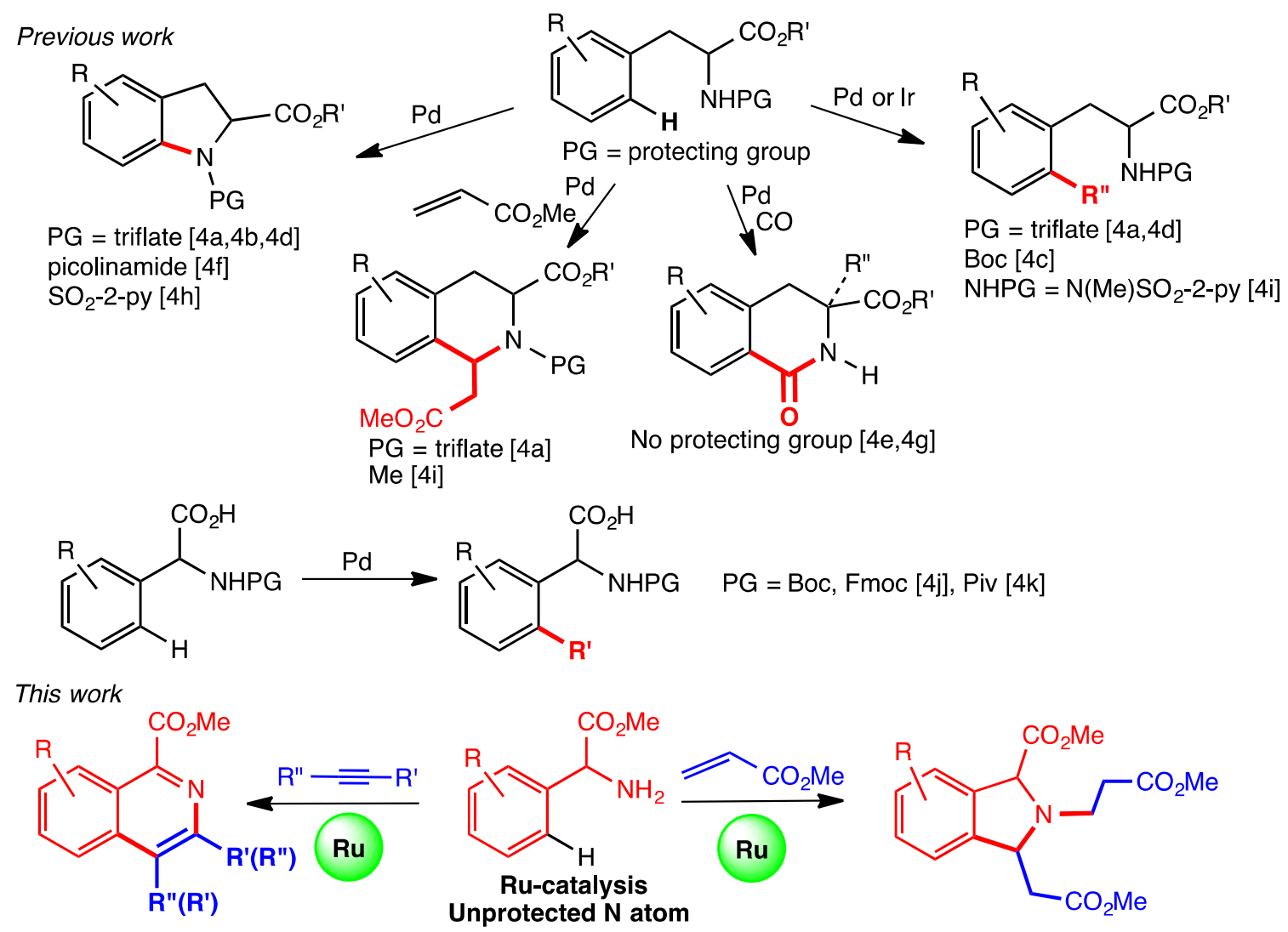

Figure 1. Functionalization of amino acids and present contribution

All the examples reported up to now share some common features. First of all, the reported processes use expensive Pd- and Ir-salts. It would be highly desirable to develop a catalytic method using a less expensive metal, such as ruthenium, while retaining the versatility and high activity of its more expensive counterparts [5]. In addition, with the exception of Pd-catalyzed carbonylations [4e,4g], the amino acid always has the nitrogen atom protected with a variety of functional groups, either monodentate or chelate. The presence of the protecting group is required in order for the reaction to 
Revised manuscript. Article published in Journal of Molecular Catalysis A: Chemical 2017, 426, 407-418. (http://dx.doi.org/10.1016/j.molcata.2016.06.026)

take place. This fact limits the reactivity of the substrate and introduces additional synthetic steps to protect, and then deprotect, the $\mathrm{N}$ atom. In this context, the reactivity of $\mathrm{N}$-protected phenylglycinates has been studied in our group. These derivatives show a higher reactivity than the $\mathrm{N}$-unprotected ones, and the $\mathrm{C}-\mathrm{H}$ bond activation step has a much lower energy barrier [6]. N-unprotected species are less prone to be activated and, therefore, their functionalization is still a great challenge.

A close inspection of the literature reveals that Ru-complexes have not so far been used as catalysts for the $\mathrm{C}-\mathrm{H}$ functionalization of amino acids, despite their well-known activity in this type of reactions [7]. For example, we have reported catalytic oxidative couplings between N-unprotected primary amines and alkynes using $\mathrm{Ru}(\mathrm{II})$ complexes to give a wide variety of $\mathrm{N}$-containing heterocycles [8]. Therefore, it is reasonable to assume that $\mathrm{Ru}(\mathrm{II})$ derivatives are very good candidates to efficiently catalyze the functionalization of amino acids avoiding the mandatory protection at the $\mathrm{N}$ atom. In the present contribution we report the results obtained in the Ru-catalyzed oxidative coupling of $\mathrm{N}$-unprotected amino acids with alkynes and olefins.

\section{Experimental}

\subsection{General methods}

Solvents were used as received from commercial sources. They were not distilled nor subjected to additional purification. All reactions were carried out without protecting atmosphere. Column liquid chromatography was performed on aluminum oxide 90 neutral $(50-200 \mu \mathrm{m})$ or silica gel $(70-230$ $\mu \mathrm{m})$. Melting points were determined on a Gallenkamp apparatus. ${ }^{1} \mathrm{H}$ and ${ }^{13} \mathrm{C}$ NMR spectra were recorded in $\mathrm{CDCl}_{3}$ solutions at $25^{\circ} \mathrm{C}$ on Bruker AV300, ARX300 or AV400 spectrometers $(\delta$ in ppm and $\mathrm{J}$ in $\mathrm{Hz}$ ) at a ${ }^{1} \mathrm{H}$ NMR operating frequency of 300.13 or $400.13 \mathrm{MHz}$, respectively. ${ }^{1} \mathrm{H}$ and ${ }^{13} \mathrm{C}$ NMR spectra were referenced using the solvent signal as an internal standard. The full assignment of ${ }^{1} \mathrm{H}$ NMR peaks was performed through standard 2D ${ }^{1} \mathrm{H}-\mathrm{COSY}$ and selective 1D ${ }^{1} \mathrm{H}-\mathrm{NOESY}$ experiments. Typical mixing times in the case of selective 1D-NOESY experiments were 1 or $2 \mathrm{~s}$, as a function of the irradiated signal. These values of optimized mixing times were set equal to the longitudinal relaxation time $\mathrm{T} 1$, which in turn was determined using the inversion-recovery sequence. Once the ${ }^{1} \mathrm{H}$ NMR signals were fully assigned, the corresponding ${ }^{13} \mathrm{C}$ NMR peaks were identified using standard ${ }^{1} \mathrm{H}-{ }^{13} \mathrm{C}$ edited-HSQC and ${ }^{1} \mathrm{H}-{ }^{13} \mathrm{C}$ HMBC 2D-experiments. ESI $\left(\mathrm{ESI}^{+}\right)$mass spectra were recorded using an Esquire 3000 ion-trap mass spectrometer (Bruker Daltonic GmbH) equipped with a standard ESI/APCI source. Samples were introduced by direct infusion with a syringe pump. Nitrogen served both as the nebulizer gas and the dry gas. HRMS and ESI $\left(\mathrm{ESI}^{+}\right)$mass spectra were recorded using an MicroToF Q, API-Q-ToF ESI with a mass range from 20 to $3000 \mathrm{~m} / \mathrm{z}$ and mass resolution 15000 (FWHM). Some products were purified using a Kugelrohr distillation apparatus 
Revised manuscript. Article published in Journal of Molecular Catalysis A: Chemical 2017, 426, 407-418. (http://dx.doi.org/10.1016/j.molcata.2016.06.026)

model Buchi B-585. The syntheses of the ruthenium catalyst $\left[\left[\mathrm{Ru}(\mu-\mathrm{Cl}) \mathrm{Cl}\left(\eta^{6}-p \text {-cymene }\right)\right]_{2}[9 \mathrm{a}]\right.$, (3nitrophenyl)glycine [9b] and methyl arylglycinate hydrochlorides $\mathbf{1 d}$ and 1e [9c], were carried out following published procedures. The spectral data were compared with the literature data to confirm their structures. Other starting phenylglycines, alkynes and alkenes were purchased from commercial sources and used as received.

\subsection{Synthesis of phenylglycine methyl ester hydrochlorides $\mathbf{1 b}, \mathbf{1 c}$, and $\mathbf{1 f}$}

\subsubsection{Synthesis of methyl (3-nitrophenyl)glycinate hydrochloride (1b)}

Thionyl chloride $(1.10 \mathrm{~mL}, 15.08 \mathrm{mmol})$ was added dropwise to an ice-cooled suspension of (3nitrophenyl)glycine $(1.39 \mathrm{~g}, 7.07 \mathrm{mmol})$ in dry methanol $(18 \mathrm{~mL})$. The resulting solution was stirred at room temperature for $24 \mathrm{~h}$. Then, the solvent was concentrated under vacuum and the residue was lyophilized to afford a pale orange solid (1.66 g, $6.71 \mathrm{mmol}, 95 \%$ yield). Mp 178-180 ${ }^{\circ} \mathrm{C}$; ${ }^{1} \mathrm{H}$ NMR $\left(\mathrm{D}_{2} \mathrm{O}, 400 \mathrm{MHz}\right)(\delta, \mathrm{ppm})$ 8.40-8.33 (m, 2H, $\left.\mathrm{C}_{6} \mathrm{H}_{4}\right), 7.91-7.86\left(\mathrm{~m}, 1 \mathrm{H}, \mathrm{C}_{6} \mathrm{H}_{4}\right), 7.78-7.72(\mathrm{~m}, 1 \mathrm{H}$, $\left.\mathrm{C}_{6} \mathrm{H}_{4}\right), 5.49$ (s, $\left.1 \mathrm{H}, \mathrm{CH}\right), 3.83\left(\mathrm{~s}, 3 \mathrm{H}, \mathrm{CH}_{3}\right) ;{ }^{13} \mathrm{C} \mathrm{NMR}\left(\mathrm{D}_{2} \mathrm{O}, 100 \mathrm{MHz}\right)(\delta, \mathrm{ppm}) 168.6(\mathrm{C}), 148.2(\mathrm{C})$, $134.6(\mathrm{CH}), 132.5(\mathrm{C}), 130.9(\mathrm{CH}), 125.2(\mathrm{CH}), 123.2(\mathrm{CH}), 55.5\left(\mathrm{CH}_{3}\right), 54.1(\mathrm{CH})$; HRMS (ESITOF) calc. for $\left[\mathrm{C}_{9} \mathrm{H}_{11} \mathrm{~N}_{2} \mathrm{O}_{4}\right]^{+}$requires 211.0713, found 211.0705 .

\subsubsection{Synthesis of methyl (4-chlorophenyl)glycinate hydrochloride (1c)}

A solution of 4-chlorobenzaldehyde $(4.79 \mathrm{~g}, 34.08 \mathrm{mmol})$ in methanol $(10 \mathrm{~mL})$ was added dropwise under vigorous stirring to a solution of $\mathrm{KCN}(2.48 \mathrm{~g}, 38.08 \mathrm{mmol})$ and $\mathrm{NH}_{4} \mathrm{Cl}(2.04 \mathrm{~g}, 38.08 \mathrm{mmol})$ in water $(10 \mathrm{~mL})$. The reaction mixture was vigorously stirred at room temperature for $2 \mathrm{~h}$. Then, water $(25 \mathrm{~mL})$ was added and the resulting mixture was extracted with toluene $(25 \mathrm{~mL})$. The aqueous layer was discarded and the toluene phase was extracted with $6 \mathrm{~N}$ aqueous $\mathrm{HCl}(3 \times 10 \mathrm{~mL})$. The combined aqueous phase was refluxed for $24 \mathrm{~h}$. After that, the reaction mixture was evaporated and the resulting residue was suspended in water. The $\mathrm{pH}$ was adjusted to 6-7 by means of the addition of small portions of $\mathrm{Na}_{2} \mathrm{CO}_{3}$ to precipitate the amino acid. The (4-chlorophenyl)glycine was filtered and dried (2.16 g, $11.62 \mathrm{mmol}, 34 \%$ yield) and used in the next step without further purification.

Thionyl chloride (1.79 mL, $24.59 \mathrm{mmol})$ was added dropwise to an ice-cooled suspension of (4chlorophenyl)glycine $(2.16 \mathrm{~g}, 11.62 \mathrm{mmol})$ in dry methanol $(29 \mathrm{~mL})$. The resulting solution was stirred at room temperature for $24 \mathrm{~h}$. Then, the solvent was concentrated under vacuum and the residue was lyophilized to afford a white solid (2.55 g, $10.80 \mathrm{mmol}, 93 \%$ yield). Mp 196-198 ${ }^{\circ} \mathrm{C}$ (lit. 194-197 $\left.{ }^{\circ} \mathrm{C}[10]\right)$; ${ }^{1} \mathrm{H}$ NMR $\left(\mathrm{D}_{2} \mathrm{O}, 400 \mathrm{MHz}\right)(\delta, \mathrm{ppm})$ 7.54-7.50 (m, 2H, $\left.\mathrm{C}_{6} \mathrm{H}_{4}\right), 7.46-7.41(\mathrm{~m}, 2 \mathrm{H}$, $\left.\mathrm{C}_{6} \mathrm{H}_{4}\right), 5.30(\mathrm{~s}, 1 \mathrm{H}, \mathrm{CH}), 3.81\left(\mathrm{~s}, 3 \mathrm{H}, \mathrm{CH}_{3}\right) ;{ }^{13} \mathrm{C} \mathrm{NMR}\left(\mathrm{D}_{2} \mathrm{O}, 100 \mathrm{MHz}\right)(\delta, \mathrm{ppm}) 169.2(\mathrm{C}), 135.9(\mathrm{C})$, 
Revised manuscript. Article published in Journal of Molecular Catalysis A: Chemical 2017, 426, 407-418. (http://dx.doi.org/10.1016/j.molcata.2016.06.026)

$129.7(2 \times \mathrm{CH}), 129.6(2 \times \mathrm{CH}), 129.5(\mathrm{C}), 55.7\left(\mathrm{CH}_{3}\right), 53.9(\mathrm{CH})$; HRMS (ESI-TOF) calc. for $\left[\mathrm{C}_{9} \mathrm{H}_{11} \mathrm{ClNO}_{2}\right]^{+}$requires 200.0473, found 200.0464 .

\subsubsection{Synthesis of methyl (4-methylphenyl)glycinate hydrochloride (1f)}

To a mixture of 4-methylbenzaldehyde $(1.18 \mathrm{~mL}, 10.00 \mathrm{mmol})$ and benzhydrylamine $(2.07 \mathrm{~mL}, 12.00$ mmol) trimethylsilyl cyanide $(1.50 \mathrm{~mL}, 12.00 \mathrm{mmol})$ was added dropwise. The reaction mixture was vigorously stirred at room temperature for 30 minutes. Then, the mixture was evaporated to remove the unreacted trimethylsilyl cyanide and the resulting residue was purified by column chromatography (eluent: hexane/Et $2 \mathrm{O} 9: 1)$ to afford a colourless oil (2.34 g, $7.48 \mathrm{mmol}, 75 \%$ yield). Spectroscopic data are in agreement with those described in literature [11].

A solution of the protected aminonitrile $(2.34 \mathrm{~g}, 7.48 \mathrm{mmol})$ in a $1: 1$ mixture of concentrated $\mathrm{HCl}$ and trifluoroacetic acid $(16 \mathrm{~mL})$ was stirred in a sealed tube at $80^{\circ} \mathrm{C}$ for $18 \mathrm{~h}$. After that, the reaction mixture was partitioned between water and ether. The organic phase was discarded and the aqueous phase was washed with ether. Then, the $\mathrm{pH}$ was adjusted to 6-7 by means of the addition of small portions of $\mathrm{Na}_{2} \mathrm{CO}_{3}$ to precipitate the amino acid. The (4-methylphenyl)glycine was filtered and dried (823 mg, $4.98 \mathrm{mmol}, 67 \%$ yield) and used in the next step without further purification.

Thionyl chloride (768 $\square \mathrm{L}, 10.46 \mathrm{mmol}$ ) was added dropwise to an ice-cooled suspension of (4methylphenyl)glycine ( $823 \mathrm{~g}, 4.98 \mathrm{mmol})$ in dry methanol $(13 \mathrm{~mL})$. The resulting solution was stirred at room temperature for $24 \mathrm{~h}$. Then, the solvent was concentrated under vacuum and the residue was lyophilized to afford a white solid (1.05 g, 4.88 mmol, 98\% yield). Mp 190-192 ${ }^{\circ} \mathrm{C}$ (lit. $192-193{ }^{\circ} \mathrm{C}$ [12]); ${ }^{1} \mathrm{H}$ NMR (MeOD, $\left.400 \mathrm{MHz}\right)(\delta, \mathrm{ppm}) 7.37-7.28$ (m, 4H, $\left.\mathrm{C}_{6} \mathrm{H}_{4}\right), 5.14$ (s, 1H, CH), 3.80 (s, 3H, $\left.\mathrm{CH}_{3}\right), 2.37$ (s, 3H, $\left.\mathrm{CH}_{3}\right) ;{ }^{13} \mathrm{C} \mathrm{NMR}(\mathrm{MeOD}, 100 \mathrm{MHz})(\delta, \mathrm{ppm}) 170.2(\mathrm{C}), 141.7(\mathrm{C}), 131.2(2 \times \mathrm{CH})$, $130.3(\mathrm{C}), 129.1(2 \times \mathrm{CH}), 57.3\left(\mathrm{CH}_{3}\right), 53.9(\mathrm{CH}), 21.2\left(\mathrm{CH}_{3}\right)$; HRMS (ESI-TOF) calc. for $\left[\mathrm{C}_{10} \mathrm{H}_{14} \mathrm{NO}_{2}\right]^{+}$requires 180.1019 , found 180.1016 .

\subsection{General synthesis of methyl isoquinoline-1-carboxylate derivatives 3 (3aa-3ae and $\mathbf{3 b a}-\mathbf{3} \mathbf{f a}$ )}

The corresponding phenylglycine methyl ester hydrochloride $(1.5 \mathrm{mmol})$ was first dissolved in a saturated solution of $\mathrm{KHCO}_{3}$ in water $(2 \mathrm{~mL})$ and stirred until bubbling ceased. Then, the solution was extracted with $\mathrm{CH}_{2} \mathrm{Cl}_{2}(3 \times 10 \mathrm{~mL})$, the organic layer was separated and dried over $\mathrm{MgSO}_{4}$ and then evaporated to dryness, in order to afford the pure phenylglycine methyl ester derivative 1a-f.

The phenylglycine methyl ester derivative $(1 \mathrm{mmol})$ and the alkyne $\mathbf{2 a - e}(2 \mathrm{mmol})$ were added to a suspension of $\left[\mathrm{Ru}(p \text {-cymene }) \mathrm{Cl}_{2}\right]_{2}(61.2 \mathrm{mg}, 0.1 \mathrm{mmol}), \mathrm{NaOAc}(164.1 \mathrm{~g}, 2.0 \mathrm{mmol}), \mathrm{K}\left[\mathrm{PF}_{6}\right]$ (18.7 $\mathrm{mg}, \quad 0.1 \mathrm{mmol})$ and $N$-fluoro-2,4,6-trimethylpyridium triflate $(289.3 \mathrm{mg}, 1 \mathrm{mmol})$ in HFIP (hexafluoroisopropanol, $3 \mathrm{~mL}$ ) into a Young's flask. The solution was stirred and heated at $60{ }^{\circ} \mathrm{C}$ for 
Revised manuscript. Article published in Journal of Molecular Catalysis A: Chemical 2017, 426, 407-418. (http://dx.doi.org/10.1016/j.molcata.2016.06.026)

$24 \mathrm{~h}$. After the reaction time was completed, the solvent was removed to dryness. The resulting solid residue was dissolved in $\mathrm{CH}_{2} \mathrm{Cl}_{2}$, filtered over neutral alumina and eluted first with $100 \mathrm{~mL}$ of $\mathrm{CH}_{2} \mathrm{Cl}_{2}$ affording a yellow solution. Evaporation of the yellow solution to dryness gave the corresponding products $\mathbf{3}$, although impure. Pure compounds $\mathbf{3}$ were obatined by further purification by column chromatography on silica gel eluting with mixtures of hexane/diethylether, and/or by Kugelrohr distillation, as specified on each particular case.

In the case of compound 3ab further elution with $50 \mathrm{~mL}$ of $\mathrm{CH}_{2} \mathrm{Cl}_{2} / \mathrm{MeOH}$ 95:5 afforded a brown solution. The brown solution contained very small amounts of organometallic product 3ab.OM, which was purified by further column chromatography using ethyl acetate/hexane (90:10 to 50:50) and crystallized as reported in Section 2.8. The small amount of crystalline 3ab.OM obtained in different batches precluded its full spectroscopic characterization.

\subsection{Characterization of products type 3}

2.4.1. Methyl 3,4-diethylisoquinoline-1-carboxylate (3aa): dark yellow oil (177 mg, 73\%). Purified by Kugelrohr distillation at $80{ }^{0} \mathrm{C}$ for 90 minutes. ${ }^{1} \mathrm{H} \mathrm{NMR}\left(\mathrm{CDCl}_{3}, 300 \mathrm{MHz}\right)(\delta, \mathrm{ppm}) 8.52(\mathrm{dd}, 1 \mathrm{H}$, $\left.\mathrm{C}_{6} \mathrm{H}_{4},{ }^{3} \mathrm{~J}_{\mathrm{HH}}=8.5,{ }^{4} \mathrm{~J}_{\mathrm{HH}}=1.4 \mathrm{~Hz}\right), 7.94\left(\mathrm{dd}, 1 \mathrm{H}, \mathrm{C}_{6} \mathrm{H}_{4},{ }^{3} \mathrm{~J}_{\mathrm{HH}}=8.7,{ }^{4} \mathrm{~J}_{\mathrm{HH}}=1.0 \mathrm{~Hz}\right), 7.59\left(\mathrm{~m}, 1 \mathrm{H}, \mathrm{C}_{6} \mathrm{H}_{4}\right)$, $7.47\left(\mathrm{~m}, 1 \mathrm{H}, \mathrm{C}_{6} \mathrm{H}_{4}\right), 3.98\left(\mathrm{~s}, 3 \mathrm{H}, \mathrm{CH}_{3}\right), 2.99\left(\mathrm{~m}, 4 \mathrm{H}, \mathrm{CH}_{2} \mathrm{CH}_{3}\right), 1.27\left(\mathrm{t}, 3 \mathrm{H}, \mathrm{CH}_{2} \mathrm{CH}_{3},{ }^{3} \mathrm{~J}_{\mathrm{HH}}=7.6 \mathrm{~Hz}\right)$, $1.21\left(\mathrm{t}, 3 \mathrm{H}, \mathrm{CH}_{2} \mathrm{CH}_{3},{ }^{3} \mathrm{~J}_{\mathrm{HH}}=7.6 \mathrm{~Hz}\right) ;{ }^{13} \mathrm{C} \mathrm{NMR}\left(\mathrm{CDCl}_{3}, 75 \mathrm{MHz}\right)(\delta, \mathrm{ppm}) 167.0(\mathrm{C}), 153.1(\mathrm{C})$, $146.9(\mathrm{C}) 135.8(\mathrm{C}), 133.1(\mathrm{C}), 130.1(\mathrm{CH}), 126.8(\mathrm{CH}), 126.7(\mathrm{CH}), 125.1(\mathrm{C}), 123.1(\mathrm{CH}), 52.9$ $\left(\mathrm{CH}_{3}\right), 28.4\left(\mathrm{CH}_{2}\right), 21.1\left(\mathrm{CH}_{2}\right), 14.7\left(\mathrm{CH}_{3}\right), 15.0\left(\mathrm{CH}_{3}\right)$; HRMS (ESI-TOF) calc. for $\left[\mathrm{C}_{15} \mathrm{H}_{17} \mathrm{NNaO}_{2}\right]^{+}$ requires 266.1151 , found 266.1178 .

2.4.2. Methyl 3,4-diphenylisoquinoline-1-carboxylate (3ab): pale yellow solid (136 mg, 40\%). Purified by column chromatography, elution with hexane/diethyl ether (90/10 to 50/50). ${ }^{1} \mathrm{H}$ NMR $\left(\mathrm{CDCl}_{3}, 300 \mathrm{MHz}\right)(\delta, \mathrm{ppm}) 8.63\left(\mathrm{~m}, 1 \mathrm{H}, \mathrm{C}_{6} \mathrm{H}_{4}\right), 7.58\left(\mathrm{~m}, 3 \mathrm{H}, \mathrm{C}_{6} \mathrm{H}_{4}\right), 7.28(\mathrm{~m}, 5 \mathrm{H}, \mathrm{Ph}), 7.13(\mathrm{~m}, 5 \mathrm{H}$, $\mathrm{Ph}), 4.02$ (s, 3H, $\left.\mathrm{CH}_{3}\right) ;{ }^{13} \mathrm{C} \mathrm{NMR}\left(\mathrm{CDCl}_{3}, 75 \mathrm{MHz}\right)(\delta, \mathrm{ppm}) 166.7$ (C), $149.6(\mathrm{C}), 148.5(\mathrm{C}), 140.0$ (C), $137.0(\mathrm{C}), 136.8(\mathrm{C}), 133.9(\mathrm{C}), 131.0(\mathrm{CH}), 130.6(\mathrm{CH}), 130.4(\mathrm{CH}), 128.4(\mathrm{CH}), 128.0(\mathrm{CH})$, $127.7(\mathrm{CH}), 127.7(\mathrm{CH}), 127.4(\mathrm{CH}), 126.2(\mathrm{CH}), 126.1(\mathrm{CH}), 125.2(\mathrm{C}), 53.0\left(\mathrm{CH}_{3}\right)$; HRMS (ESITOF) calc. for $\left[\mathrm{C}_{23} \mathrm{H}_{17} \mathrm{NNaO}_{2}\right]^{+}$requires 362.1151 , found 362.1164 .

2.4.3. Methyl 3-methyl-4-propylisoquinoline-1-carboxylate (3ac1) and methyl 4-methyl-3propylisoquinoline-1-carboxylate (3ac2): compounds $3 \mathbf{a c 1}$ and 3ac2 were separated and purified by column chromatography, elution with hexane/diethyl ether (90/10 to 50/50). 3ac1 eluted first.

3ac1: orange solid (41 mg, 17\%). Traces of subproducts were removed by Kugelrohr distillation at $80{ }^{0} \mathrm{C}$ for 90 minutes. ${ }^{1} \mathrm{H}$ NMR $\left(\mathrm{CDCl}_{3}, 300 \mathrm{MHz}\right)\left(\delta\right.$, ppm) $8.62\left(\mathrm{~d}, 1 \mathrm{H}, \mathrm{C}_{6} \mathrm{H}_{4},{ }^{3} \mathrm{~J}_{\mathrm{HH}}=8.6 \mathrm{~Hz}\right), 7.96$ 
Revised manuscript. Article published in Journal of Molecular Catalysis A: Chemical 2017, 426, 407-418. (http://dx.doi.org/10.1016/j.molcata.2016.06.026)

$\left(\mathrm{dd}, 1 \mathrm{H}, \mathrm{C}_{6} \mathrm{H}_{4},{ }^{3} \mathrm{~J}_{\mathrm{HH}}=8.7,{ }^{4} \mathrm{~J}_{\mathrm{HH}}=1.1 \mathrm{~Hz}\right), 7.64\left(\mathrm{ddd}, 1 \mathrm{H}, \mathrm{C}_{6} \mathrm{H}_{4},{ }^{3} \mathrm{~J}_{\mathrm{HH}}=8.5,{ }^{3} \mathrm{~J}_{\mathrm{HH}}=6.8,{ }^{4} \mathrm{~J}_{\mathrm{HH}}=1.3 \mathrm{~Hz}\right)$, $7.51\left(\mathrm{ddd}, 1 \mathrm{H}, \mathrm{C}_{6} \mathrm{H}_{4},{ }^{3} \mathrm{~J}_{\mathrm{HH}}=8.5,{ }^{3} \mathrm{~J}_{\mathrm{HH}}=6.8,{ }^{4} \mathrm{~J}_{\mathrm{HH}}=1.2 \mathrm{~Hz}\right), 4.01\left(\mathrm{~s}, 3 \mathrm{H}, \mathrm{O}-\mathrm{CH}_{3}\right), 2.99(\mathrm{~m}, 2 \mathrm{H}$, $\left.\mathrm{CH}_{2} \mathrm{CH}_{2} \mathrm{CH}_{3}\right), 2.71\left(\mathrm{~s}, 3 \mathrm{H}, \mathrm{CH}_{3}\right), 1.60\left(\mathrm{~m}, 2 \mathrm{H}, \mathrm{CH}_{2} \mathrm{CH}_{2} \mathrm{CH}_{3}\right), 1.02\left(\mathrm{t}, 3 \mathrm{H}, \mathrm{CH}_{2} \mathrm{CH}_{2} \mathrm{CH}_{3},{ }^{3} \mathrm{~J}_{\mathrm{HH}}=7.4\right.$ $\mathrm{Hz}) ;{ }^{13} \mathrm{C} \mathrm{NMR}\left(\mathrm{CDCl}_{3}, 75 \mathrm{MHz}\right)(\delta, \mathrm{ppm}) 166.6(\mathrm{C}), 148.2$ (C), $146.0(\mathrm{C}), 136.1$ (C), $133.0(\mathrm{C})$, $130.2(\mathrm{CH}), 127.0(\mathrm{CH}), 126.7(\mathrm{CH}), 125.3(\mathrm{C}), 53.0\left(\mathrm{CH}_{3}\right), 123.1(\mathrm{CH}), 30.5\left(\mathrm{CH}_{2}\right), 23.2\left(\mathrm{CH}_{2}\right)$, $22.4\left(\mathrm{CH}_{3}\right), 14.5\left(\mathrm{CH}_{3}\right)$. HRMS (ESI-TOF) calc. for $\left[\mathrm{C}_{15} \mathrm{H}_{17} \mathrm{NNaO}_{2}\right]^{+}$requires 266.1151 , found 266.1154. 3ac2: yellow solid (61 mg, 25\%). Traces of subproducts were removed by Kugelrohr distillation at $80{ }^{0} \mathrm{C}$ for 90 minutes. ${ }^{1} \mathrm{H}$ NMR $\left(\mathrm{CDCl}_{3}, 300 \mathrm{MHz}\right)(\delta, \mathrm{ppm}) 8.55\left(\mathrm{ddd}, 1 \mathrm{H}, \mathrm{C}_{6} \mathrm{H}_{4},{ }^{3} \mathrm{~J}_{\mathrm{HH}}\right.$ $\left.=8.6,{ }^{4} \mathrm{~J}_{\mathrm{HH}}=1.3,{ }^{5} \mathrm{~J}_{\mathrm{HH}}=0.7 \mathrm{~Hz}\right), 7.96\left(\mathrm{ddd}, 1 \mathrm{H}, \mathrm{C}_{6} \mathrm{H}_{4},{ }^{3} \mathrm{~J}_{\mathrm{HH}}=8.6,{ }^{4} \mathrm{~J}_{\mathrm{HH}}=1.4,{ }^{5} \mathrm{~J} \mathrm{HH}=0.7 \mathrm{~Hz}\right), 7.63(\mathrm{ddd}$, $\left.1 \mathrm{H}, \mathrm{C}_{6} \mathrm{H}_{4},{ }^{3} \mathrm{~J}_{\mathrm{HH}}=7.6,{ }^{3} \mathrm{~J}_{\mathrm{HH}}=6.7,{ }^{4} \mathrm{~J}_{\mathrm{HH}}=1.8 \mathrm{~Hz}\right), 7.51\left(\mathrm{~m}, 1 \mathrm{H}, \mathrm{C}_{6} \mathrm{H}_{4}\right), 4.00\left(\mathrm{~s}, 3 \mathrm{H}, \mathrm{O}-\mathrm{CH}_{3}\right), 2.97(\mathrm{~m}$, $\left.2 \mathrm{H}, \mathrm{CH}_{2} \mathrm{CH}_{2} \mathrm{CH}_{3}\right), 2.60\left(\mathrm{~s}, 3 \mathrm{H}, \mathrm{CH}_{3}\right), 1.70\left(\mathrm{~m}, 2 \mathrm{H}, \mathrm{CH}_{2} \mathrm{CH}_{2} \mathrm{CH}_{3}\right), 0.95\left(\mathrm{t}, 3 \mathrm{H},{ }^{3} \mathrm{~J}_{\mathrm{HH}}=7.3 \mathrm{~Hz}\right.$,

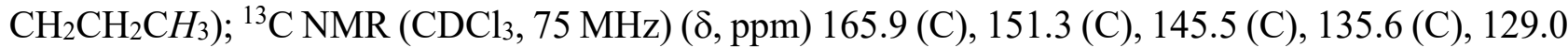
$(\mathrm{CH}), 126.5(\mathrm{C}), 125.9(\mathrm{CH}), 125.5(\mathrm{CH}), 123.7(\mathrm{C}), 122.3(\mathrm{CH}), 51.91\left(\mathrm{CH}_{3}\right), 36.9\left(\mathrm{CH}_{2}\right), 22.2$ $\left(\mathrm{CH}_{2}\right), 13.2\left(\mathrm{CH}_{3}\right), 13.1\left(\mathrm{CH}_{3}\right)$. HRMS (ESI-TOF) calc. for $\left[\mathrm{C}_{15} \mathrm{H}_{17} \mathrm{NNaO}_{2}\right]^{+}$requires 266.1151, found 266.1162 .

2.4.4. Methyl 3-methyl-4-phenylisoquinoline-1-carboxylate (3ad1) and methyl 4-methyl-3phenylisoquinoline-1-carboxylate (3ad2): compounds 3ad1 and 3ad2 were obtained as a mixture in 1:4 molar ratio. Compound 3ad1 could not be obtained in pure form (it was always contaminated with 3ad2), while 3ad2 was obtained in pure form by column chromatography by elution with hexane/diethyl ether (90/10 to 50/50). Traces of subproducts were removed by Kugelrohr distillation at $80{ }^{\circ} \mathrm{C}$ for 90 minutes. Compound 3ad2: dark yellow oil (141 mg, 51\%). ${ }^{1} \mathrm{H} \mathrm{NMR}\left(\mathrm{CDCl}_{3}, 300\right.$ $\mathrm{MHz})(\delta, \mathrm{ppm}) 8.64\left(\mathrm{ddd}, 1 \mathrm{H}, \mathrm{C}_{6} \mathrm{H}_{4},{ }^{3} \mathrm{~J}_{\mathrm{HH}}=8.5,{ }^{4} \mathrm{~J}_{\mathrm{HH}}=1.4,{ }^{5} \mathrm{~J}_{\mathrm{HH}}=0.7 \mathrm{~Hz}\right), 8.03\left(\mathrm{ddd}, 1 \mathrm{H}, \mathrm{C}_{6} \mathrm{H}_{4},{ }^{3} \mathrm{~J}_{\mathrm{HH}}\right.$ $\left.=8.6,{ }^{4} \mathrm{~J}_{\mathrm{HH}}=1.3,{ }^{5} \mathrm{~J}_{\mathrm{HH}}=0.7 \mathrm{~Hz}\right), 7.70\left(\mathrm{ddd}, 1 \mathrm{H}, \mathrm{C}_{6} \mathrm{H}_{4},{ }^{3} \mathrm{~J}_{\mathrm{HH}}=8.4,{ }^{3} \mathrm{~J}_{\mathrm{HH}}=6.8,{ }^{4} \mathrm{~J}_{\mathrm{HH}}=1.4 \mathrm{~Hz}\right), 7.59$ $\left(\mathrm{ddd}, 1 \mathrm{H}, \mathrm{C}_{6} \mathrm{H}_{4},{ }^{3} \mathrm{~J}_{\mathrm{HH}}=8.2,{ }^{3} \mathrm{~J}_{\mathrm{HH}}=6.8,{ }^{4} \mathrm{~J}_{\mathrm{HH}}=1.2 \mathrm{~Hz}\right), 7.51(\mathrm{~m}, 2 \mathrm{H}, \mathrm{Ph}), 7.37(\mathrm{~m}, 3 \mathrm{H}, \mathrm{Ph}), 3.97(\mathrm{~s}$,

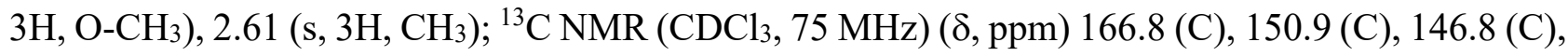
$140.5(\mathrm{C}), 137.1(\mathrm{C}), 130.5(\mathrm{CH}), 130.1(\mathrm{CH}), 128.2(\mathrm{CH}), 128.1(\mathrm{C}), 127.9(\mathrm{CH}), 127.8(\mathrm{CH}), 127.0$ $(\mathrm{CH}) 125.1(\mathrm{C}), 124.0(\mathrm{CH}), 52.9\left(\mathrm{CH}_{3}\right), 16.1\left(\mathrm{CH}_{3}\right)$. HRMS (ESI-TOF) calc. for $\left[\mathrm{C}_{18} \mathrm{H}_{15} \mathrm{NNaO}_{2}\right]^{+}$ requires 300.0995 , found 300.1005 .

2.4.5. Methyl 4-(tert-butyl)-3-methylisoquinoline-1-carboxylate (3ae1), methyl 3-(tert-butyl)-4methylisoquinoline-1-carboxylate (3ae2) and methyl 1-amino-2-(tert-butyl)-3-methyl-1H-indene-1carboxylate (3ae3): compounds 3ae1, 3ae2 and 3ae3 were separated and purified by column chromatography. Elution with hexane/diethyl ether (90/10 to 50/50) gave pure 3ae1 as the product which eluted first, then $\mathbf{3 a e} 3$ and finally $\mathbf{3 a e 2}$. 
Revised manuscript. Article published in Journal of Molecular Catalysis A: Chemical 2017, 426, 407-418. (http://dx.doi.org/10.1016/j.molcata.2016.06.026)

3ae1: yellow solid (39 mg, 15\%). ${ }^{1} \mathrm{H}$ NMR $\left(\mathrm{CDCl}_{3}, 300 \mathrm{MHz}\right)(\delta, \mathrm{ppm}) 8.51\left(\mathrm{dd}, 1 \mathrm{H}, \mathrm{C}_{6} \mathrm{H}_{4},{ }^{3} \mathrm{~J}_{\mathrm{HH}}=\right.$ $\left.8.3,{ }^{4} \mathrm{~J}_{\mathrm{HH}}=1.1 \mathrm{~Hz}\right), 8.32\left(\mathrm{dd}, 1 \mathrm{H}, \mathrm{C}_{6} \mathrm{H}_{4},{ }^{3} \mathrm{~J}_{\mathrm{HH}}=8.8,{ }^{4} \mathrm{~J}_{\mathrm{HH}}=1.2 \mathrm{~Hz}\right), 7.52\left(\mathrm{ddd}, 1 \mathrm{H}, \mathrm{C}_{6} \mathrm{H}_{4},{ }^{3} \mathrm{~J}_{\mathrm{HH}}=8.8\right.$, $\left.{ }^{3} \mathrm{~J}_{\mathrm{HH}}=6.7,{ }^{4} \mathrm{~J}_{\mathrm{HH}}=1.1 \mathrm{~Hz}\right), 7.43\left(\mathrm{ddd}, 1 \mathrm{H}, \mathrm{C}_{6} \mathrm{H}_{4},{ }^{3} \mathrm{~J}_{\mathrm{HH}}=8.3,{ }^{3} \mathrm{~J}_{\mathrm{HH}}=6.7,{ }^{4} \mathrm{~J}_{\mathrm{HH}}=1.2 \mathrm{~Hz}\right), 4.00(\mathrm{~s}, 3 \mathrm{H}$, $\left.\mathrm{OCH}_{3}\right), 2.81\left(\mathrm{~s}, 3 \mathrm{H}, \mathrm{CH}_{3}\right), 1.64\left(\mathrm{~s}, 9 \mathrm{H}, \mathrm{C}\left(\mathrm{CH}_{3}\right)_{3}\right) ;{ }^{13} \mathrm{C} \mathrm{NMR}\left(\mathrm{CDCl}_{3}, 75 \mathrm{MHz}\right)(\delta, \mathrm{ppm}) 166.6(\mathrm{C})$, $147.5(\mathrm{C}), 145.5(\mathrm{C}), 140.0(\mathrm{C}), 136.9(\mathrm{C}), 127.8(\mathrm{CH}), 126.3(\mathrm{CH}), 126.1(\mathrm{CH}), 125.9(\mathrm{CH}), 125.6$ (C), $53.0\left(\mathrm{CH}_{3}\right), 37.1(\mathrm{C}), 33.3\left(\mathrm{CH}_{3}\right), 28.9\left(\mathrm{CH}_{3}\right)$. HRMS (ESI-TOF) calc. for $\left[\mathrm{C}_{16} \mathrm{H}_{19} \mathrm{NNaO}_{2}\right]^{+}$ requires 280.1308, found 280.1297. 3ae2: yellow solid (64 mg, 25\%). ${ }^{1} \mathrm{H} \mathrm{NMR}\left(\mathrm{CDCl}_{3}, 300 \mathrm{MHz}\right)$ $(\delta, \mathrm{ppm}) 8.56\left(\mathrm{ddd}, 1 \mathrm{H}, \mathrm{C}_{6} \mathrm{H}_{4},{ }^{3} \mathrm{~J}_{\mathrm{HH}}=8.5,{ }^{4} \mathrm{~J}_{\mathrm{HH}}=1.3,{ }^{5} \mathrm{~J}_{\mathrm{HH}}=0.7 \mathrm{~Hz}\right), 8.01\left(\mathrm{ddd}, 1 \mathrm{H}, \mathrm{C}_{6} \mathrm{H}_{4},{ }^{3} \mathrm{~J}_{\mathrm{HH}}=8.7\right.$, $\left.{ }^{4} \mathrm{~J}_{\mathrm{HH}}=1.2 \mathrm{~Hz},{ }^{5} \mathrm{~J}_{\mathrm{HH}}=0.7 \mathrm{~Hz}\right), 7.62\left(\mathrm{ddd}, 1 \mathrm{H}, \mathrm{C}_{6} \mathrm{H}_{4},{ }^{3} \mathrm{~J}_{\mathrm{HH}}=8.7,{ }^{3} \mathrm{~J}_{\mathrm{HH}}=6.8,{ }^{4} \mathrm{~J}_{\mathrm{HH}}=1.3 \mathrm{~Hz}\right), 7.51(\mathrm{ddd}$, $\left.1 \mathrm{H}, \mathrm{C}_{6} \mathrm{H}_{4},{ }^{3} \mathrm{~J}_{\mathrm{HH}}=8.6,{ }^{3} \mathrm{~J}_{\mathrm{HH}}=6.8,{ }^{4} \mathrm{~J}_{\mathrm{HH}}=1.2 \mathrm{~Hz}\right), 3.99\left(\mathrm{~s}, 3 \mathrm{H}, \mathrm{O}-\mathrm{CH}_{3}\right), 2.78\left(\mathrm{~s}, 3 \mathrm{H}, \mathrm{CH}_{3}\right), 1.50(\mathrm{~s}, 9 \mathrm{H}$, $\left.\mathrm{C}\left(\mathrm{CH}_{3}\right)_{3}\right) ;{ }^{13} \mathrm{C} \mathrm{NMR}\left(\mathrm{CDCl}_{3}, 75 \mathrm{MHz}\right)(\delta, \mathrm{ppm}) 167.3(\mathrm{C}), 157.6(\mathrm{C}), 144.5(\mathrm{C}), 137.9(\mathrm{C}), 129.6$ $(\mathrm{CH}), 127.9(\mathrm{C}), 126.9(\mathrm{CH}), 126.2(\mathrm{CH}), 124.5(\mathrm{C}), 123.2(\mathrm{CH}), 52.6\left(\mathrm{CH}_{3}\right), 38.8(\mathrm{C}), 30.8\left(\mathrm{CH}_{3}\right)$, $16.6\left(\mathrm{CH}_{3}\right)$. HRMS (ESI-TOF) calc. for $\left[\mathrm{C}_{16} \mathrm{H}_{19} \mathrm{NNaO}_{2}\right]^{+}$requires 280.1308 , found 280.1285. 3ae3: yellow oil (104 mg, 40\%). ${ }^{1} \mathrm{H}$ NMR $\left(\mathrm{CDCl}_{3}, 300 \mathrm{MHz}\right)\left(\delta\right.$, ppm) $7.18\left(\mathrm{~m}, 1 \mathrm{H}, \mathrm{C}_{6} \mathrm{H}_{4}\right), 7.07$ (m, 3H, $\left.\mathrm{C}_{6} \mathrm{H}_{4}\right), 3.52$ (s, 3H, $\left.\mathrm{OCH}_{3}\right), 2.14$ (s, 3H, $\left.\mathrm{CH}_{3}\right), 2.02$ (sbroad, $\left.2 \mathrm{H}, \mathrm{NH}_{2}\right), 1.28\left(\mathrm{~s}, 9 \mathrm{H}, \mathrm{C}\left(\mathrm{CH}_{3}\right)_{3}\right) ;{ }^{13} \mathrm{C} \mathrm{NMR}$ $\left(\mathrm{CDCl}_{3}, 75 \mathrm{MHz}\right)(\delta, \mathrm{ppm}) 175.4(\mathrm{C}), 151.6(\mathrm{C}), 147.3(\mathrm{C}), 145.7(\mathrm{C}), 135.2(\mathrm{C}), 128.4(\mathrm{CH}), 125.7$ $(\mathrm{CH}), 119.8(\mathrm{CH}), 118.5(\mathrm{CH}), 72.6(\mathrm{C}), 52.4\left(\mathrm{CH}_{3}\right), 34.2(\mathrm{C}), 30.2\left(\mathrm{CH}_{3}\right), 13.1\left(\mathrm{CH}_{3}\right)$. HRMS (ESITOF) calc. for $\left[\mathrm{C}_{16} \mathrm{H}_{22} \mathrm{NO}_{2}\right]^{+}$requires 260.1645 , found 260.1645 .

2.4.6. Methyl 3,4-diethyl-7-nitroisoquinoline-1-carboxylate (3ba): white solid (118 $\mathrm{mg}, 41 \%)$. Purified by Kugelrohr distillation at $80{ }^{\circ} \mathrm{C}$ for 90 minutes, and then column chromatography: elution with hexane/diethyl ether (90/10 to 50/50). ${ }^{1} \mathrm{H}$ NMR $\left(\mathrm{CDCl}_{3}, 300 \mathrm{MHz}\right)(\delta, \mathrm{ppm}) 9.61\left(\mathrm{~d}, 1 \mathrm{H}, \mathrm{C}_{6} \mathrm{H}_{3}\right.$, $\left.{ }^{4} \mathrm{~J}_{\mathrm{HH}}=2.4 \mathrm{~Hz}\right), 8.38\left(\mathrm{dd}, 1 \mathrm{H}, \mathrm{C}_{6} \mathrm{H}_{3},{ }^{3} \mathrm{~J}_{\mathrm{HH}}=9.4,{ }^{4} \mathrm{~J}_{\mathrm{HH}}=2.4 \mathrm{~Hz}\right), 8.11\left(\mathrm{~d}, 1 \mathrm{H}, \mathrm{C}_{6} \mathrm{H}_{3},{ }^{3} \mathrm{~J}_{\mathrm{HH}}=9.4 \mathrm{~Hz}\right), 4.06$ $\left(\mathrm{s}, 3 \mathrm{H}, \mathrm{CH}_{3}\right), 3.11\left(\mathrm{q}, 2 \mathrm{H}, \mathrm{CH}_{2} \mathrm{CH}_{3},{ }^{3} \mathrm{~J}_{\mathrm{HH}}=7.6 \mathrm{~Hz}\right), 3.04\left(\mathrm{q}, 2 \mathrm{H}, \mathrm{CH}_{2} \mathrm{CH}_{3},{ }^{3} \mathrm{~J}_{\mathrm{HH}}=7.6 \mathrm{~Hz}\right), 1.32(\mathrm{t}, 3 \mathrm{H}$,

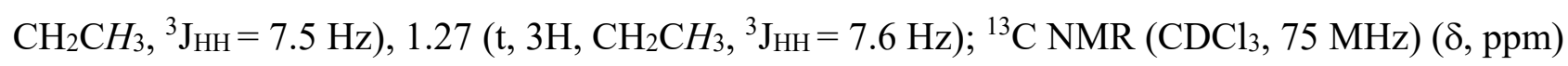
165.9 (C), 157.1 (C), 148.1 (C), 145.9 (C), 138.1 (C), 133.7 (C), 125.3 (CH), 124.0 (C), 124.0 (CH), $123.3(\mathrm{CH}), 53.4\left(\mathrm{CH}_{3}\right), 28.6\left(\mathrm{CH}_{2}\right), 21.4\left(\mathrm{CH}_{2}\right), 15.0\left(\mathrm{CH}_{3}\right), 14.4\left(\mathrm{CH}_{3}\right)$. HRMS (ESI-TOF) calc. for $\left[\mathrm{C}_{15} \mathrm{H}_{16} \mathrm{~N}_{2} \mathrm{NaO}_{4}\right]^{+}$requires 311.1002, found 311.0998.

2.4.7. Methyl 6-chloro-3,4-diethylisoquinoline-1-carboxylate (3ca): yellow oil (164 mg, 60\%). Purified by column chromatography: elution with hexane/diethyl ether (90/10 to 50/50). ${ }^{1} \mathrm{H}$ NMR $\left(\mathrm{CDCl}_{3}, 300 \mathrm{MHz}\right)(\delta, \mathrm{ppm}) 8.54\left(\mathrm{dd}, 1 \mathrm{H}, \mathrm{C}_{6} \mathrm{H}_{3},{ }^{3} \mathrm{~J}_{\mathrm{HH}}=9.2,{ }^{5} \mathrm{~J}_{\mathrm{HH}}=0.6 \mathrm{~Hz}\right), 7.88\left(\mathrm{dd}, 1 \mathrm{H}, \mathrm{C}_{6} \mathrm{H}_{3},{ }^{4} \mathrm{~J}_{\mathrm{HH}}\right.$ $\left.=2.1,{ }^{5} \mathrm{~J}_{\mathrm{HH}}=0.6 \mathrm{~Hz}\right), 7.39\left(\mathrm{dd}, 1 \mathrm{H}, \mathrm{C}_{6} \mathrm{H}_{3},{ }^{3} \mathrm{~J}_{\mathrm{HH}}=9.2,{ }^{4} \mathrm{~J}_{\mathrm{HH}}=2.1 \mathrm{~Hz}\right), 3.98\left(\mathrm{~s}, 3 \mathrm{H}, \mathrm{OCH}_{3}\right), 2.96(\mathrm{~m}, 4 \mathrm{H}$, 
Revised manuscript. Article published in Journal of Molecular Catalysis A: Chemical 2017, 426, 407-418. (http://dx.doi.org/10.1016/j.molcata.2016.06.026)

$\left.\mathrm{CH}_{2}\right), 1.26\left(\mathrm{t}, 3 \mathrm{H},{ }^{3} \mathrm{~J}_{\mathrm{HH}}=7.6 \mathrm{~Hz}\right), 1.20\left(\mathrm{t}, 3 \mathrm{H},{ }^{3} \mathrm{~J}_{\mathrm{HH}}=7.6 \mathrm{~Hz}\right) ;{ }^{13} \mathrm{C} \mathrm{NMR}\left(\mathrm{CDCl}_{3}, 75 \mathrm{MHz}\right)(\delta, \mathrm{ppm})$ $166.6(\mathrm{C}), 154.3(\mathrm{C}), 146.5(\mathrm{C}), 136.8(\mathrm{C}), 136.6(\mathrm{C}), 132.4(\mathrm{C}), 128.6(\mathrm{CH}), 127.8(\mathrm{CH}), 123.4(\mathrm{C})$, $122.2(\mathrm{CH}), 53.0\left(\mathrm{CH}_{3}\right), 28.5\left(\mathrm{CH}_{2}\right), 21.2\left(\mathrm{CH}_{2}\right), 14.9\left(\mathrm{CH}_{3}\right), 14.6\left(\mathrm{CH}_{3}\right)$. HRMS (ESI-TOF) calc. for $\left[\mathrm{C}_{15} \mathrm{H}_{16} \mathrm{ClNNaO}_{2}\right]^{+}$requires 300.0764 , found 300.0762 .

2.4.8. Methyl 7-bromo-3,4-diethylisoquinoline-1-carboxylate (3da1) and methyl 5-bromo-3,4diethylisoquinoline-1-carboxylate (3da2): not separated. Obtained as a colorless oil (106 mg, 33\% mixture: 25 and 8\%). Purified by column chromatography: elution with hexane/diethyl ether (90/10 to 50/50). HRMS (ESI-TOF) calc. for $\left[\mathrm{C}_{15} \mathrm{H}_{16} \mathrm{BrNNaO}_{2}\right]^{+}$requires 344.0257 , found 344.0266. 3da1 (major isomer): ${ }^{1} \mathrm{H} \mathrm{NMR}\left(\mathrm{CDCl}_{3}, 300 \mathrm{MHz}\right)(\delta, \mathrm{ppm}) 8.82\left(\mathrm{dd}, 1 \mathrm{H}, \mathrm{C}_{6} \mathrm{H}_{3},{ }^{4} \mathrm{~J}_{\mathrm{HH}}=2.0,{ }^{5} \mathrm{~J}_{\mathrm{HH}}=0.5 \mathrm{~Hz}\right)$, $7.83\left(\mathrm{dd}, 1 \mathrm{H}, \mathrm{C}_{6} \mathrm{H}_{3},{ }^{3} \mathrm{~J}_{\mathrm{HH}}=9.2,{ }^{5} \mathrm{~J}_{\mathrm{HH}}=0.5 \mathrm{~Hz}\right), 7.69\left(\mathrm{dd}, 1 \mathrm{H}, \mathrm{C}_{6} \mathrm{H}_{3},{ }^{3} \mathrm{~J}_{\mathrm{HH}}=9.2,{ }^{4} \mathrm{~J}_{\mathrm{HH}}=2.0 \mathrm{~Hz}\right), 4.00(\mathrm{~s}$,

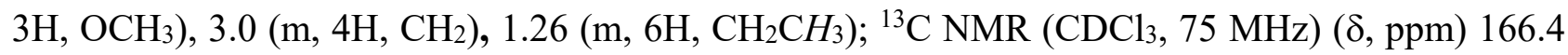
(C), 153.6(C), $145.3(\mathrm{C}), 134.4(\mathrm{C}), 133.6(\mathrm{CH}), 133.6(\mathrm{C}), 129.0(\mathrm{CH}), 126.3(\mathrm{C}), 125.0(\mathrm{CH}), 121.4$ (C), $53.1\left(\mathrm{CH}_{3}\right), 28.4\left(\mathrm{CH}_{2}\right), 21.2\left(\mathrm{CH}_{2}\right), 15.0\left(\mathrm{CH}_{3}\right), 14.6\left(\mathrm{CH}_{3}\right), 3 \mathrm{da} 2$ (minor isomer): ${ }^{1} \mathrm{H} \mathrm{NMR}$ $\left(\mathrm{CDCl}_{3}, 300 \mathrm{MHz}\right)(\delta, \mathrm{ppm}) 8.34\left(\mathrm{dd}, 1 \mathrm{H}, \mathrm{C}_{6} \mathrm{H}_{3},{ }^{3} \mathrm{~J}_{\mathrm{HH}}=8.5,{ }^{4} \mathrm{~J}_{\mathrm{HH}}=1.3 \mathrm{~Hz}\right), 7.98\left(\mathrm{dd}, 1 \mathrm{H}, \mathrm{C}_{6} \mathrm{H}_{3},{ }^{3} \mathrm{~J}_{\mathrm{HH}}\right.$ $\left.=7.5,{ }^{4} \mathrm{~J}_{\mathrm{HH}}=1.3 \mathrm{~Hz}\right), 7.25\left(\mathrm{dd}, 1 \mathrm{H}, \mathrm{C}_{6} \mathrm{H}_{3},{ }^{3} \mathrm{~J}_{\mathrm{HH}}=8.5,{ }^{3} \mathrm{~J}_{\mathrm{HH}}=7.5 \mathrm{~Hz}\right), 4.00\left(\mathrm{~s}, 3 \mathrm{H}, \mathrm{OCH}_{3}\right), 3.51(\mathrm{q}$,

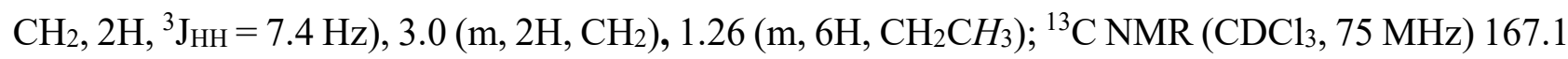
(C), $156.2(\mathrm{C}), 148.7(\mathrm{C}), 138.5(\mathrm{CH}), 134.4(\mathrm{C}), 133.0(\mathrm{C}), 126.9(\mathrm{CH}), 126.8$ (C), $117.9(\mathrm{C}), 53.1$ $\left(\mathrm{CH}_{3}\right), 29.1\left(\mathrm{CH}_{2}\right), 22.1\left(\mathrm{CH}_{2}\right), 16.8\left(\mathrm{CH}_{3}\right), 14.7\left(\mathrm{CH}_{3}\right)$.

2.4.9. Methyl 3,4-diethyl-6-methoxyisoquinoline-1-carboxylate (3ea): yellow solid (197 mg, 72\%). Purified by column chromatography: elution with hexane/diethyl ether (90/10 to 50/50). ${ }^{1} \mathrm{H}$ NMR $\left(\mathrm{CDCl}_{3}, 400 \mathrm{MHz}\right)(\delta, \mathrm{ppm}) \quad 8.56\left(\mathrm{~m}, 1 \mathrm{H}, \mathrm{C}_{6} \mathrm{H}_{3}\right), 7.20\left(\mathrm{~m}, 2 \mathrm{H}, \mathrm{C}_{6} \mathrm{H}_{3}\right), 4.06\left(\mathrm{~s}, 3 \mathrm{H}, \mathrm{C}(=\mathrm{O}) \mathrm{OCH}_{3}\right)$, $3.95\left(\mathrm{~s}, 3 \mathrm{H}, \mathrm{OCH}_{3}\right), 3.05\left(\mathrm{~m}, 4 \mathrm{H}, \mathrm{CH}_{2} \mathrm{CH}_{3}\right), 1.35$ (t, $\left.3 \mathrm{H}, \mathrm{CH}_{2} \mathrm{CH}_{3},{ }^{3} \mathrm{~J}_{\mathrm{HH}}=7.6 \mathrm{~Hz}\right), 1.30\left(\mathrm{t}, 3 \mathrm{H}, \mathrm{CH}_{2} \mathrm{CH}_{3}\right.$, $\left.{ }^{3} \mathrm{~J}_{\mathrm{HH}}=7.6 \mathrm{~Hz}\right) ;{ }^{13} \mathrm{C} \mathrm{NMR}\left(\mathrm{CDCl}_{3}, 100 \mathrm{MHz}\right)(\delta, \mathrm{ppm}) 167.1(\mathrm{C}), 160.6(\mathrm{C}), 153.5(\mathrm{C}), 146.2(\mathrm{C})$ $137.9(\mathrm{C}), 131.9(\mathrm{C}), 128.6(\mathrm{CH}), 121.0(\mathrm{C}), 119.5(\mathrm{CH}), 101.2(\mathrm{CH}), 55.3\left(\mathrm{CH}_{3}\right), 52.8\left(\mathrm{CH}_{3}\right), 28.6$ $\left(\mathrm{CH}_{2}\right), 21.3\left(\mathrm{CH}_{2}\right), 14.8\left(\mathrm{CH}_{3}\right), 14.4\left(\mathrm{CH}_{3}\right)$. HRMS (ESI-TOF) calc. for $\left[\mathrm{C}_{16} \mathrm{H}_{19} \mathrm{NNaO}_{3}\right]^{+}$requires 296.1257 , found 296.1253 .

2.4.10. Methyl 3,4-diethyl-6-methylisoquinoline-1-carboxylate (3fa): yellow oil (193 mg, 75\%). Purified by column chromatography: elution with hexane/diethyl ether (90/10 to $50 / 50) .{ }^{1} \mathrm{H}$ NMR $\left(\mathrm{CDCl}_{3}, 300 \mathrm{MHz}\right)(\delta, \mathrm{ppm}) 8.44\left(\mathrm{~d}, 1 \mathrm{H}, \mathrm{C}_{6} \mathrm{H}_{3},{ }^{3} \mathrm{~J}_{\mathrm{HH}}=8.8 \mathrm{~Hz}\right), 7.69\left(\mathrm{~s}, 1 \mathrm{H}, \mathrm{C}_{6} \mathrm{H}_{3}\right), 7.31\left(\mathrm{dd}, 1 \mathrm{H}, \mathrm{C}_{6} \mathrm{H}_{3}\right.$, $\left.{ }^{3} \mathrm{~J}_{\mathrm{HH}}=8.8,{ }^{4} \mathrm{~J}_{\mathrm{HH}}=1.6 \mathrm{~Hz}\right), 3.98\left(\mathrm{~d}, 3 \mathrm{H}, \mathrm{CH}_{3},{ }^{4} \mathrm{~J}_{\mathrm{HH}}=1.2 \mathrm{~Hz}\right), 2.99\left(\mathrm{~m}, 4 \mathrm{H}, \mathrm{CH}_{2} \mathrm{CH}_{3}\right), 2.47(\mathrm{~s}, 3 \mathrm{H}$, $\left.\mathrm{OCH}_{3}\right), 1.25\left(\mathrm{~m}, 6 \mathrm{H}, \mathrm{CH}_{2} \mathrm{CH}_{3}\right) ;{ }^{13} \mathrm{C} \mathrm{NMR}\left(\mathrm{CDCl}_{3}, 75 \mathrm{MHz}\right)(\delta, \mathrm{ppm}) 167.1(\mathrm{C}), 153.1(\mathrm{C}), 146.5(\mathrm{C})$, 
Revised manuscript. Article published in Journal of Molecular Catalysis A: Chemical 2017, 426, 407-418. (http://dx.doi.org/10.1016/j.molcata.2016.06.026)

$140.3(\mathrm{C}), 136.1(\mathrm{C}), 132.6(\mathrm{C}), 129.1(\mathrm{CH}), 126.5(\mathrm{CH}), 123.6(\mathrm{C}), 122.0(\mathrm{CH}), 52.9\left(\mathrm{CH}_{3}\right), 28.5$ $\left(\mathrm{CH}_{2}\right), 22.4\left(\mathrm{CH}_{3}\right), 21.1\left(\mathrm{CH}_{2}\right), 15.0\left(\mathrm{CH}_{3}\right), 14.8\left(\mathrm{CH}_{3}\right)$. HRMS (ESI-TOF) calc. for $\mathrm{C}_{16} \mathrm{H}_{19} \mathrm{NNaO}_{2}$ requires 280.1308 , found 280.1298 .

\subsection{General synthesis of methyl isoindoline-1-carboxylate derivatives $\mathbf{5}$ (5aa-5fa)}

The corresponding phenylglycine methyl ester hydrochloride $(1.5 \mathrm{mmol})$ was first dissolved in a saturated solution of $\mathrm{KHCO}_{3}$ in water $(10 \mathrm{~mL})$ and stirred until bubbling ceased. Then, the solution was extracted with $\mathrm{CH}_{2} \mathrm{Cl}_{2}(3 \times 10 \mathrm{~mL})$, the organic layer was separated and dried over $\mathrm{MgSO}_{4}$ and then evaporated to dryness, in order to afford the pure phenylglycine methyl ester derivative.

The phenylglycine methyl ester derivative 1a-f $(1 \mathrm{mmol})$ and alkene $4 \mathbf{a}(2 \mathrm{mmol})$ were added to a suspension of [Ru(p-cymene)Cl $]_{2}(61.2 \mathrm{mg}, 0.1 \mathrm{mmol}), \mathrm{NaOAc}(164.1 \mathrm{~g}, 2.0 \mathrm{mmol}), \mathrm{K}[\mathrm{PF} 6]$ (18.7 $\mathrm{mg}, \quad 0.1 \mathrm{mmol})$ and $N$-fluoro-2,4,6-trimethylpyridium triflate $(289.3 \mathrm{mg}, 1 \mathrm{mmol})$ in hexafluoroisopropanol $(3 \mathrm{~mL})$ into a Young's flask. The solution was stirred and heated at $60{ }^{\circ} \mathrm{C}$ for $24 \mathrm{~h}$. After the reaction time was completed, the solvent was removed to dryness. After the reaction time was completed, the solvent was removed to dryness. The resulting solid residue was dissolved in $\mathrm{CH}_{2} \mathrm{Cl}_{2}$, filtered over neutral alumina and eluted with $100 \mathrm{~mL}$ of $\mathrm{CH}_{2} \mathrm{Cl}_{2}$, affording a yellow solution. The yellow solution contained the corresponding product 5 contaminated with other reaction side products. It was evaporated to dryness and the residue was further purified by column chromatography on silica gel eluting with mixtures of hexane/diethylether, and/or by Kugelrohr distillation, as specified on each particular case.

\subsection{Characterization of products type 5}

2.6.1. Methyl 3-(2-methoxy-2-oxoethyl)-2-(3-methoxy-3-oxopropyl)isoindoline-1-carboxylate (5aa): orange oil (44 mg, 13\%). 5aa was obtained as a mixture of diastereoisomers in 1:0.4 molar ratio. It was purified by column chromatography: elution with hexane/diethyl ether (90/10 to 50/50). HRMS (ESI-TOF) calc. for $\left[\mathrm{C}_{17} \mathrm{H}_{21} \mathrm{NNaO}_{6}\right]^{+}$requires 358.1261, found 358.1270. Major diastereoisomer: ${ }^{1} \mathrm{H} \mathrm{NMR}\left(\mathrm{CDCl}_{3}, 300 \mathrm{MHz}\right)(\delta, \mathrm{ppm}) 7.15\left(\mathrm{~m}, 4 \mathrm{H}, \mathrm{C}_{6} \mathrm{H}_{4}\right), 4.65(\mathrm{~s}, 1 \mathrm{H}, \mathrm{CH}), 4.57\left(\mathrm{t}, 1 \mathrm{H}, \mathrm{CH},{ }^{3} \mathrm{~J}_{\mathrm{HH}}=\right.$ $6.2 \mathrm{~Hz}), 3.67\left(\mathrm{~s}, 3 \mathrm{H}, \mathrm{OCH}_{3}\right), 3.67\left(\mathrm{~s}, 3 \mathrm{H}, \mathrm{OCH}_{3}\right), 3.60\left(\mathrm{~s}, 3 \mathrm{H}, \mathrm{OCH}_{3}\right), 3.24\left(\mathrm{~m}, 1 \mathrm{H}, \mathrm{N}-\mathrm{CH}_{2} \mathrm{CH}_{2}\right), 3.11$ $\left(\mathrm{m}, 1 \mathrm{H}, \mathrm{N}-\mathrm{CH}_{2} \mathrm{CH}_{2}\right), 2.85\left(\mathrm{dd}, 1 \mathrm{H}, \mathrm{CH}-\mathrm{CH}_{2},{ }^{2} \mathrm{~J}_{\mathrm{HH}}=15.9,{ }^{3} \mathrm{~J}_{\mathrm{HH}}=5.8 \mathrm{~Hz}\right), 2.65\left(\mathrm{dd}, 1 \mathrm{H}, \mathrm{CH}-\mathrm{CH}_{2},{ }^{2} \mathrm{~J}_{\mathrm{HH}}\right.$ $\left.=15.9,{ }^{3} \mathrm{~J}_{\mathrm{HH}}=5.8 \mathrm{~Hz}\right), 2.46\left(\mathrm{~m}, 2 \mathrm{H}, \mathrm{N}-\mathrm{CH}_{2} \mathrm{CH}_{2}\right) ;{ }^{13} \mathrm{C} \mathrm{NMR}\left(\mathrm{CDCl}_{3}, 75 \mathrm{MHz}\right)(\delta, \mathrm{ppm}) 173.0(\mathrm{C})$, $172.6(\mathrm{C}), 172.4(\mathrm{C}), 142.1(\mathrm{C}), 136.9(\mathrm{C}), 128.4(\mathrm{CH}), 127.7(\mathrm{CH}), 122.7(\mathrm{CH}), 122.5(\mathrm{CH}), 71.3$ $(\mathrm{CH}), 65.9(\mathrm{CH}), 52.3\left(\mathrm{CH}_{3}\right), 51.6\left(\mathrm{CH}_{3}\right), 51.6\left(\mathrm{CH}_{3}\right), 49.5\left(\mathrm{CH}_{2}\right), 41.9\left(\mathrm{CH}_{2}\right), 33.45\left(\mathrm{CH}_{2}\right)$. Minor diastereoisomer: ${ }^{1} \mathrm{H} \mathrm{NMR}\left(\mathrm{CDCl}_{3}, 300 \mathrm{MHz}\right)(\delta, \mathrm{ppm}) 7.15\left(\mathrm{~m}, 4 \mathrm{H}, \mathrm{C}_{6} \mathrm{H}_{4}\right), 4.92\left(\mathrm{~d}, 1 \mathrm{H}, \mathrm{CH},{ }^{4} \mathrm{~J}_{\mathrm{HH}}=\right.$ $2.6 \mathrm{~Hz},), 4.79(\mathrm{~m}, 1 \mathrm{H}, \mathrm{CH}), 3.64\left(\mathrm{~s}, 3 \mathrm{H}, \mathrm{OCH}_{3}\right), 3.62\left(\mathrm{~s}, 3 \mathrm{H}, \mathrm{OCH}_{3}\right), 3.60\left(\mathrm{~s}, 3 \mathrm{H}, \mathrm{OCH}_{3}\right), 3.24(\mathrm{~m}$, 
Revised manuscript. Article published in Journal of Molecular Catalysis A: Chemical 2017, 426, 407-418. (http://dx.doi.org/10.1016/j.molcata.2016.06.026)

$\left.1 \mathrm{H}, \mathrm{N}-\mathrm{CH}_{2} \mathrm{CH}_{2}\right), 3.11\left(\mathrm{~m}, 1 \mathrm{H}, \mathrm{N}-\mathrm{CH}_{2} \mathrm{CH}_{2}\right), 2.85\left(\mathrm{dd}, 1 \mathrm{H}, \mathrm{CH}-\mathrm{CH}_{2},{ }^{2} \mathrm{~J}_{\mathrm{HH}}=15.9,{ }^{3} \mathrm{~J}_{\mathrm{HH}}=5.8 \mathrm{~Hz}\right), 2.65$ $\left(\mathrm{dd}, 1 \mathrm{H}, \mathrm{CH}-\mathrm{CH}_{2},{ }^{2} \mathrm{~J}_{\mathrm{HH}}=15.9,{ }^{3} \mathrm{~J}_{\mathrm{HH}}=5.8 \mathrm{~Hz}\right), 2.46\left(\mathrm{~m}, 2 \mathrm{H}, \mathrm{N}-\mathrm{CH}_{2} \mathrm{CH}_{2}\right) ;{ }^{13} \mathrm{C} \mathrm{NMR}\left(\mathrm{CDCl}_{3}, 75 \mathrm{MHz}\right)$

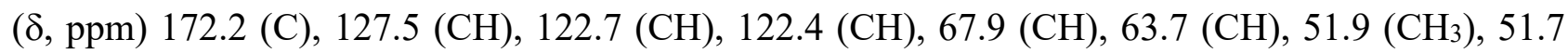
$\left(\mathrm{CH}_{3}\right), 51.7\left(\mathrm{CH}_{3}\right), 43.1\left(\mathrm{CH}_{2}\right), 39.1\left(\mathrm{CH}_{2}\right), 34.12\left(\mathrm{CH}_{2}\right)$. Some $\mathrm{C}$ were not observed for the minor isomer due to partial overlap with signals of the major isomer.

2.6.2. Methyl 3-(2-methoxy-2-oxoethyl)-2-(3-methoxy-3-oxopropyl)-6-nitroisoindoline-1carboxylate (5ba): yellow oil (103 $\mathrm{mg}, 27 \%)$. 5ba was obtained as a mixture of diastereoisomers in 1:0.3 molar ratio. The major diastereoisomer could be separated and purified by column chromatography: elution with hexane/diethyl ether (90/10 to 50/50). Major diastereoisomer: ${ }^{1} \mathrm{H}$ NMR $\left(\mathrm{CDCl}_{3}, 300 \mathrm{MHz}\right)(\delta, \mathrm{ppm}) 8.21\left(\mathrm{~m}, 2 \mathrm{H}, \mathrm{C}_{6} \mathrm{H}_{3}\right), 7.41\left(\mathrm{~m}, 1 \mathrm{H}, \mathrm{C}_{6} \mathrm{H}_{3}\right), 4.90(\mathrm{~s}, 1 \mathrm{H}, \mathrm{CH}), 4.79$ $\left(\mathrm{t}, 1 \mathrm{H}, \mathrm{CH},{ }^{3} \mathrm{~J}_{\mathrm{HH}}=6.2 \mathrm{~Hz}\right), 3.83\left(\mathrm{~s}, 3 \mathrm{H}, \mathrm{OCH}_{3}\right), 3.76\left(\mathrm{~s}, 3 \mathrm{H}, \mathrm{OCH}_{3}\right), 3.71\left(\mathrm{~s}, 3 \mathrm{H}, \mathrm{OCH}_{3}\right), 3.43(\mathrm{dt}, 1 \mathrm{H}$, $\left.\mathrm{NCH}_{2} \mathrm{CH}_{2},{ }^{2} \mathrm{~J}_{\mathrm{HH}}=14.4,{ }^{3} \mathrm{~J}_{\mathrm{HH}}=7.4 \mathrm{~Hz}\right), 3.28\left(\mathrm{dt}, 1 \mathrm{H}, \mathrm{NCH}_{2} \mathrm{CH}_{2},{ }^{2} \mathrm{~J}_{\mathrm{HH}}=13.4,{ }^{3} \mathrm{~J}_{\mathrm{HH}}=6.5 \mathrm{~Hz}\right), 3.05(\mathrm{dd}$, $\left.1 \mathrm{H}, \mathrm{CHCH}_{2},{ }^{2} \mathrm{~J}_{\mathrm{HH}}=16.3,{ }^{3} \mathrm{~J}_{\mathrm{HH}}=5.0 \mathrm{~Hz}\right), 2.80\left(\mathrm{dd}, 1 \mathrm{H}, \mathrm{CHCH}_{2},{ }^{2} \mathrm{~J}_{\mathrm{HH}}=16.3,{ }^{3} \mathrm{~J}_{\mathrm{HH}}=7.1 \mathrm{~Hz}\right), 2.62(\mathrm{t}$, $\left.2 \mathrm{H}, \mathrm{NCH}_{2} \mathrm{CH}_{2},{ }^{3} \mathrm{~J}_{\mathrm{HH}}=6.9 \mathrm{~Hz}\right) ;{ }^{13} \mathrm{C} \mathrm{NMR}\left(\mathrm{CDCl}_{3}, 75 \mathrm{MHz}\right) 172.3$ (C), 171.7 (C), 171.3 (C), 148.8 $(\mathrm{CH}), 148.2(\mathrm{CH}), 138.4(\mathrm{CH}), 124.2(\mathrm{CH}), 123.6(\mathrm{CH}), 118.6(\mathrm{CH}), 70.4(\mathrm{CH}), 65.7(\mathrm{CH}), 52.8$ $\left(\mathrm{CH}_{3}\right), 51.9\left(\mathrm{CH}_{3}\right), 51.8\left(\mathrm{CH}_{3}\right), 49.2\left(\mathrm{CH}_{2}\right), 40.7\left(\mathrm{CH}_{2}\right), 33.1\left(\mathrm{CH}_{2}\right)$. HRMS (ESI-TOF) calc. for $\left[\mathrm{C}_{17} \mathrm{H}_{20} \mathrm{~N}_{2} \mathrm{NaO}_{8}\right]^{+}$requires 403.1112 , found 403.1118 .

\subsubsection{Methyl 5-chloro-3-(2-methoxy-2-oxoethyl)-2-(3-methoxy-3-oxopropyl)isoindoline-1-} carboxylate (5ca): yellow oil (137 mg, 37\%). 5ca was obtained as a mixture of diastereoisomers in 1:0.2 molar ratio. They were separated and purified by column chromatography: elution with hexane/diethyl ether (90/10 to 50/50). The minor isomer eluted first. Major diastereoisomer: ${ }^{1} \mathrm{H}$ $\operatorname{NMR}\left(\mathrm{CDCl}_{3}, 300 \mathrm{MHz}\right)(\delta, \mathrm{ppm}) 7.16\left(\mathrm{~m}, 2 \mathrm{H}, \mathrm{C}_{6} \mathrm{H}_{3}\right), 7.11\left(\mathrm{~m}, 1 \mathrm{H}, \mathrm{C}_{6} \mathrm{H}_{3}\right), 4.60\left(\mathrm{~d}, 1 \mathrm{H}, \mathrm{CH},{ }^{4} \mathrm{~J}_{\mathrm{HH}}=\right.$ $1.3 \mathrm{~Hz}), 4.53\left(\mathrm{dd}, 1 \mathrm{H}, \mathrm{CH},{ }^{3} \mathrm{~J}_{\mathrm{HH}}=6.7,{ }^{3} \mathrm{~J}_{\mathrm{HH}}=5.8 \mathrm{~Hz}\right), 3.67\left(\mathrm{~s}, 6 \mathrm{H}, \mathrm{OCH}_{3}\right), 3.60\left(\mathrm{~s}, 3 \mathrm{H}, \mathrm{OCH}_{3}\right), 3.25$ $\left(\mathrm{m}, 2 \mathrm{H}, \mathrm{NCH}_{2} \mathrm{CH}_{2}\right), 3.08\left(\mathrm{dt}, 2 \mathrm{H}, \mathrm{NCH}_{2} \mathrm{CH}_{2},{ }^{2} \mathrm{~J}_{\mathrm{HH}}=13.4,{ }^{3} \mathrm{~J}_{\mathrm{HH}}=6.6 \mathrm{~Hz}\right), 2.84\left(\mathrm{dd}, 1 \mathrm{H}, \mathrm{CHCH}_{2},{ }^{2} \mathrm{~J}_{\mathrm{HH}}\right.$ $\left.=16.1,{ }^{3} \mathrm{~J}_{\mathrm{HH}}=5.5 \mathrm{~Hz}\right), 2.63\left(\mathrm{dd}, 1 \mathrm{H}, \mathrm{CHCH}_{2},{ }^{2} \mathrm{~J}_{\mathrm{HH}}=16.1,{ }^{3} \mathrm{~J}_{\mathrm{HH}}=6.9 \mathrm{~Hz}\right), 2.45\left(\mathrm{t}, 2 \mathrm{H}, \mathrm{NCH}_{2} \mathrm{CH}_{2}\right.$, $\left.{ }^{3} \mathrm{~J}_{\mathrm{HH}}=7.1 \mathrm{~Hz}\right) ;{ }^{13} \mathrm{C} \mathrm{NMR}\left(\mathrm{CDCl}_{3}, 75 \mathrm{MHz}\right)(\delta, \mathrm{ppm}) 172.5(\mathrm{C}), 172.5(\mathrm{C}), 172.1(\mathrm{C}), 144.1(\mathrm{C}), 135.4$ (C), $134.3(\mathrm{C}), 128.0(\mathrm{CH}), 124.0(\mathrm{CH}), 123.0(\mathrm{CH}), 70.7(\mathrm{CH}), 65.5(\mathrm{CH}), 52.4\left(\mathrm{CH}_{3}\right), 51.7\left(\mathrm{CH}_{3}\right)$, $51.6\left(\mathrm{CH}_{3}\right), 49.4\left(\mathrm{CH}_{2}\right), 41.6\left(\mathrm{CH}_{2}\right), 33.4\left(\mathrm{CH}_{2}\right)$. HRMS (ESI-TOF) calc. for $\left[\mathrm{C}_{17} \mathrm{H}_{20} \mathrm{ClNNaO}_{6}{ }^{+}\right.$ requires 392.0871, found 392.0872. Minor diastereoisomer: ${ }^{1} \mathrm{H} \mathrm{NMR}\left(\mathrm{CDCl}_{3}, 300 \mathrm{MHz}\right)(\delta, \mathrm{ppm})$ $7.15\left(\mathrm{~m}, 2 \mathrm{H}, \mathrm{C}_{6} \mathrm{H}_{3}\right), 7.13\left(\mathrm{~m}, 1 \mathrm{H}, \mathrm{C}_{6} \mathrm{H}_{3}\right), 4.91\left(\mathrm{~d}, 1 \mathrm{H}, \mathrm{CH},{ }^{4} \mathrm{~J}_{\mathrm{HH}}=2.5 \mathrm{~Hz}\right), 4.78\left(\mathrm{dt}, 1 \mathrm{H}, \mathrm{CH},{ }^{3} \mathrm{~J}_{\mathrm{HH}}=\right.$ $\left.6.3,{ }^{4} \mathrm{~J}_{\mathrm{HH}}=2.8 \mathrm{~Hz}\right), 3.66\left(\mathrm{~s}, 3 \mathrm{H}, \mathrm{OCH}_{3}\right), 3.63\left(\mathrm{~s}, 3 \mathrm{H}, \mathrm{OCH}_{3}\right), 3.61\left(\mathrm{~s}, 3 \mathrm{H}, \mathrm{OCH}_{3}\right), 3.18(\mathrm{~m}, 2 \mathrm{H}$,

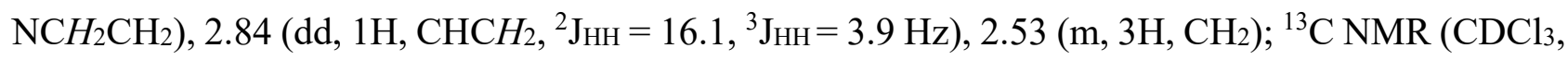
$75 \mathrm{MHz})(\delta, \mathrm{ppm}) 172.3(\mathrm{C}), 171.9(\mathrm{C}), 171.8(\mathrm{C}), 144.6(\mathrm{C}), 135.5(\mathrm{C}), 134.3(\mathrm{C}), 127.9(\mathrm{CH}), 123.6$ 
Revised manuscript. Article published in Journal of Molecular Catalysis A: Chemical 2017, 426, 407-418. (http://dx.doi.org/10.1016/j.molcata.2016.06.026)

$(\mathrm{CH}), 123.2(\mathrm{CH}), 67.2(\mathrm{CH}), 63.6(\mathrm{CH}), 52.0\left(\mathrm{CH}_{3}\right), 51.8\left(\mathrm{CH}_{3}\right), 51.7\left(\mathrm{CH}_{3}\right), 43.0\left(\mathrm{CH}_{2}\right), 38.6\left(\mathrm{CH}_{2}\right)$, $33.8\left(\mathrm{CH}_{2}\right)$. HRMS (ESI-TOF) calc. for $\left[\mathrm{C}_{17} \mathrm{H}_{20} \mathrm{ClNNaO}_{6}\right]^{+}$requires 392.0871, found 392.0859.

2.6.4. Methyl 4-bromo-3-(2-methoxy-2-oxoethyl)-2-(3-methoxy-3-oxopropyl)isoindoline-1carboxylate (5da1) and methyl 6-bromo-3-(2-methoxy-2-oxoethyl)-2-(3-methoxy-3oxopropyl)isoindoline-1-carboxylate (5da2): yellow oil (156 mg, 38\%). 5da was obtained as the mixture of the two possible regioisomers $(\mathbf{5 d a 1}: 5 \mathbf{d a 2}=0.4: 1$ molar ratio), which were not separated. The mixture was purified by column chromatography: elution with hexane/diethyl ether (90/10 to 50/50). HRMS (ESI-TOF) calc. for $\left[\mathrm{C}_{17} \mathrm{H}_{20} \mathrm{BrNNaO}_{6}\right]^{+}$requires 436.0366, found 436.0347. 5da1 (minor isomer): ${ }^{1} \mathrm{H} \mathrm{NMR}\left(\mathrm{CDCl}_{3}, 300 \mathrm{MHz}\right)(\delta, \mathrm{ppm}) 7.37\left(\mathrm{~m}, 1 \mathrm{H}, \mathrm{C}_{6} \mathrm{H}_{3}\right), 7.23\left(\mathrm{~m}, 1 \mathrm{H}, \mathrm{C}_{6} \mathrm{H}_{3}\right), 7.08$ $\left(\mathrm{m}, 1 \mathrm{H}, \mathrm{C}_{6} \mathrm{H}_{3}\right), 4.69(\mathrm{~s}, 1 \mathrm{H}, \mathrm{CH}), 4.66(\mathrm{~s}, 1 \mathrm{H}, \mathrm{CH}), 3.67$ (s, 3H, $\left.\mathrm{OCH}_{3}\right), 3.67\left(\mathrm{~s}, 3 \mathrm{H}, \mathrm{OCH}_{3}\right), 3.58(\mathrm{~s}$, $\left.3 \mathrm{H}, \mathrm{OCH}_{3}\right), 3.25\left(\mathrm{~m}, 1 \mathrm{H}, \mathrm{NCH}_{2} \mathrm{CH}_{2}\right), 3.11\left(\mathrm{~m}, 1 \mathrm{H}, \mathrm{NCH}_{2} \mathrm{CH}_{2}\right), 3.04\left(\mathrm{~m}, 1 \mathrm{H}, \mathrm{CHCH}_{2}\right), 2.76(\mathrm{dd}, 1 \mathrm{H}$,

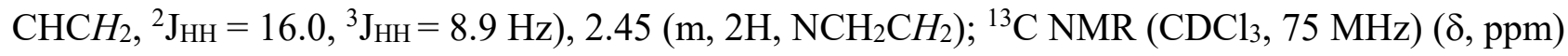
$172.6(\mathrm{C}), 172.6(\mathrm{C}), 172.3(\mathrm{C}), 141.8(\mathrm{C}), 139.7(\mathrm{C}), 132.1(\mathrm{CH}), 129.6(\mathrm{CH}), 122.4(\mathrm{CH}), 117.5$ (C), $72.2(\mathrm{CH}), 68.1(\mathrm{CH}), 52.4\left(\mathrm{CH}_{3}\right), 51.7\left(\mathrm{CH}_{3}\right), 51.6\left(\mathrm{CH}_{3}\right), 51.4\left(\mathrm{CH}_{2}\right), 40.4\left(\mathrm{CH}_{2}\right), 33.9\left(\mathrm{CH}_{2}\right)$. 5da2 (major isomer): ${ }^{1} \mathrm{H}$ NMR $\left(\mathrm{CDCl}_{3}, 300 \mathrm{MHz}\right)(\delta, \mathrm{ppm}) 7.38\left(\mathrm{~m}, 1 \mathrm{H}, \mathrm{C}_{6} \mathrm{H}_{3}\right), 7.34\left(\mathrm{~m}, 1 \mathrm{H}, \mathrm{C}_{6} \mathrm{H}_{3}\right)$, $7.00\left(\mathrm{~d}, 1 \mathrm{H}, \mathrm{C}_{6} \mathrm{H}_{3},{ }^{3} \mathrm{~J}_{\mathrm{HH}}=8.1 \mathrm{~Hz}\right), 4.64\left(\mathrm{t}, 1 \mathrm{H}, \mathrm{CH},{ }^{4} \mathrm{~J}_{\mathrm{HH}}=1.2 \mathrm{~Hz}\right), 4.51(\mathrm{~m}, 1 \mathrm{H}, \mathrm{CH}), 3.69(\mathrm{~s}, 3 \mathrm{H}$, $\left.\mathrm{OCH}_{3}\right), 3.66\left(\mathrm{~s}, 3 \mathrm{H}, \mathrm{OCH}_{3}\right), 3.60\left(\mathrm{~s}, 3 \mathrm{H}, \mathrm{OCH}_{3}\right), 3.25\left(\mathrm{dt}, 1 \mathrm{H}, \mathrm{NCH}_{2} \mathrm{CH}_{2},{ }^{2} \mathrm{~J}_{\mathrm{HH}}=13.6,{ }^{3} \mathrm{JHH}=7.5 \mathrm{~Hz}\right)$, $3.10\left(\mathrm{~m}, 1 \mathrm{H}, \mathrm{NCH}_{2} \mathrm{CH}_{2}\right), 2.85\left(\mathrm{dd}, 1 \mathrm{H}, \mathrm{CHCH}_{2},{ }^{2} \mathrm{~J}_{\mathrm{HH}}=16.0,{ }^{3} \mathrm{~J}_{\mathrm{HH}}=5.5 \mathrm{~Hz}\right), 2.62(\mathrm{dd}, 1 \mathrm{H}, \mathrm{CHCH}$,

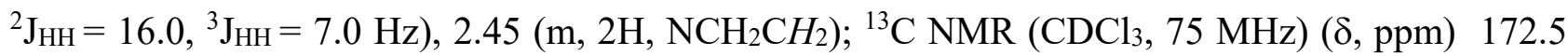
(C), $172.3(\mathrm{C}), 172.1(\mathrm{C}), 141.1(\mathrm{C}), 139.1(\mathrm{C}), 131.5(\mathrm{CH}), 126.0(\mathrm{CH}), 124.2(\mathrm{CH}), 121.5(\mathrm{C}), 70.7$ $(\mathrm{CH}), 65.5(\mathrm{CH}), 52.5\left(\mathrm{CH}_{3}\right), 51.7\left(\mathrm{CH}_{3}\right), 51.7\left(\mathrm{CH}_{3}\right), 49.3\left(\mathrm{CH}_{2}\right), 41.5\left(\mathrm{CH}_{2}\right), 33.4\left(\mathrm{CH}_{2}\right)$.

2.6.5. Methyl 5-methoxy-3-(2-methoxy-2-oxoethyl)-2-(3-methoxy-3-oxopropyl)isoindoline-1carboxylate (5ea): yellow oil (161 mg, $44 \%$ ). 5ea was obtained as a mixture of diastereoisomers in 1:0.2 molar ratio. The major diastereoisomer could be separated and purified by column chromatography: elution with hexane/diethyl ether (90/10 to 50/50). Major diastereoisomer: ${ }^{1} \mathrm{H}$ $\operatorname{NMR}\left(\mathrm{CDCl}_{3}, 300 \mathrm{MHz}\right)(\delta, \mathrm{ppm}) 7.12\left(\mathrm{~d}, 1 \mathrm{H}, \mathrm{C}_{6} \mathrm{H}_{3},{ }^{3} \mathrm{~J}_{\mathrm{HH}}=8.4 \mathrm{~Hz}\right), 6.73\left(\mathrm{dd}, 1 \mathrm{H}, \mathrm{C}_{6} \mathrm{H}_{3},{ }^{3} \mathrm{~J}_{\mathrm{HH}}=8.6\right.$, $\left.{ }^{4} \mathrm{~J}_{\mathrm{HH}}=2.4 \mathrm{~Hz}\right), 6.64\left(\mathrm{~d}, 1 \mathrm{H}, \mathrm{C}_{6} \mathrm{H}_{3},{ }^{4} \mathrm{~J}_{\mathrm{HH}}=2.3 \mathrm{~Hz}\right), 4.57(\mathrm{~s}, 1 \mathrm{H}, \mathrm{CH}), 4.52\left(\mathrm{t}, 1 \mathrm{H}, \mathrm{CH},{ }^{3} \mathrm{~J}_{\mathrm{HH}}=6.3 \mathrm{~Hz}\right)$, $3.71\left(\mathrm{~s}, 3 \mathrm{H}, \mathrm{OCH}_{3}\right), 3.67\left(\mathrm{~s}, 3 \mathrm{H}, \mathrm{OCH}_{3}\right), 3.66\left(\mathrm{~s}, 3 \mathrm{H}, \mathrm{OCH}_{3}\right), 3.60\left(\mathrm{~s}, 3 \mathrm{H}, \mathrm{OCH}_{3}\right), 3.25(\mathrm{dt}, 1 \mathrm{H}$, $\left.\mathrm{NCH}_{2} \mathrm{CH}_{2},{ }^{2} \mathrm{~J}_{\mathrm{HH}}=13.5,{ }^{3} \mathrm{~J}_{\mathrm{HH}}=7.6 \mathrm{~Hz}\right), 3.07\left(\mathrm{dt}, 1 \mathrm{H}, \mathrm{NCH}_{2} \mathrm{CH}_{2},{ }^{2} \mathrm{~J}_{\mathrm{HH}}=13.4,{ }^{3} \mathrm{~J}_{\mathrm{HH}}=6.7 \mathrm{~Hz}\right), 2.84(\mathrm{dd}$, $\left.1 \mathrm{H}, \mathrm{CH}-\mathrm{CH}_{2},{ }^{2} \mathrm{~J}_{\mathrm{HH}}=15.9,{ }^{3} \mathrm{~J}_{\mathrm{HH}}=5.8 \mathrm{~Hz}\right), 2.64\left(\mathrm{dd}, 1 \mathrm{H}, \mathrm{CHCH}_{2},{ }^{2} \mathrm{~J}_{\mathrm{HH}}=15.9,{ }^{3} \mathrm{~J}_{\mathrm{HH}}=6.8 \mathrm{~Hz}\right), 2.44(\mathrm{t}$, $\left.2 \mathrm{H}, \mathrm{NCH}_{2} \mathrm{CH}_{2},{ }^{3} \mathrm{~J}_{\mathrm{HH}}=7.2 \mathrm{~Hz}\right) ;{ }^{13} \mathrm{C} \mathrm{NMR}\left(\mathrm{CDCl}_{3}, 75 \mathrm{MHz}\right)(\delta, \mathrm{ppm}) 173.3(\mathrm{C}), 172.6(\mathrm{C}), 172.5(\mathrm{C})$, $160.17(\mathrm{C}), 143.7(\mathrm{C}), 129.0(\mathrm{C}), 123.5(\mathrm{CH}), 113.9(\mathrm{CH}), 107.7(\mathrm{CH}), 70.7(\mathrm{CH}), 65.9(\mathrm{CH}), 55.5$ 
Revised manuscript. Article published in Journal of Molecular Catalysis A: Chemical 2017, 426, 407-418. (http://dx.doi.org/10.1016/j.molcata.2016.06.026)

$\left(\mathrm{CH}_{3}\right), 52.2\left(\mathrm{CH}_{3}\right), 51.7\left(\mathrm{CH}_{3}\right), 51.6\left(\mathrm{CH}_{3}\right), 49.6\left(\mathrm{CH}_{2}\right), 41.9\left(\mathrm{CH}_{2}\right), 33.4\left(\mathrm{CH}_{2}\right)$. HRMS (ESI-TOF) calc. for $\left[\mathrm{C}_{18} \mathrm{H}_{23} \mathrm{NNaO}_{7}\right]^{+}$requires 388.1367 , found 388.1371

2.6.6. Methyl 3-(2-methoxy-2-oxoethyl)-2-(3-methoxy-3-oxopropyl)-5-methylisoindoline-1carboxylate (5fa): yellow oil (231 mg, 66\%). 5fa was obtained as a mixture of diastereoisomers in 1:0.1 molar ratio. The major diastereoisomer could be separated and purified by column chromatography: elution with hexane/diethyl ether (90/10 to 50/50). Major diastereoisomer: ${ }^{1} \mathrm{H}$ $\mathrm{NMR}\left(\mathrm{CDCl}_{3}, 300 \mathrm{MHz}\right)(\delta, \mathrm{ppm}) 7.10\left(\mathrm{~d}, 1 \mathrm{H}, \mathrm{C}_{6} \mathrm{H}_{3},{ }^{3} \mathrm{~J}_{\mathrm{HH}}=7.8 \mathrm{~Hz}\right), 6.99\left(\mathrm{dd}, 1 \mathrm{H}, \mathrm{C}_{6} \mathrm{H}_{3},{ }^{3} \mathrm{~J}_{\mathrm{HH}}=7.8\right.$, $\left.{ }^{4} \mathrm{~J}_{\mathrm{HH}}=0.6 \mathrm{~Hz}\right), 6.90\left(\mathrm{~s}, 1 \mathrm{H}, \mathrm{C}_{6} \mathrm{H}_{3}\right), 4.59(\mathrm{~s}, 1 \mathrm{H}, \mathrm{CH}), 4.52\left(\mathrm{t}, 1 \mathrm{H}, \mathrm{CH},{ }^{3} \mathrm{~J}_{\mathrm{HH}}=6.2 \mathrm{~Hz}\right), 3.67(\mathrm{~s}, 3 \mathrm{H}$, $\left.\mathrm{OCH}_{3}\right), 3.65\left(\mathrm{~s}, 3 \mathrm{H}, \mathrm{OCH}_{3}\right), 3.59\left(\mathrm{~s}, 3 \mathrm{H}, \mathrm{OCH}_{3}\right), 3.25\left(\mathrm{dt}, 1 \mathrm{H}, \mathrm{NCH}_{2} \mathrm{CH}_{2},{ }^{2} \mathrm{~J}_{\mathrm{HH}}=13.5,{ }^{3} \mathrm{~J}_{\mathrm{HH}}=7.6 \mathrm{~Hz}\right)$, $3.07\left(\mathrm{dt}, 1 \mathrm{H}, \mathrm{NCH}_{2} \mathrm{CH}_{2},{ }^{2} \mathrm{~J}_{\mathrm{HH}}=13.4,{ }^{3} \mathrm{~J}_{\mathrm{HH}}=6.7 \mathrm{~Hz}\right), 2.83\left(\mathrm{dd}, \mathrm{CHCH}_{2},{ }^{2} \mathrm{~J}_{\mathrm{HH}}=15.9,{ }^{3} \mathrm{~J}_{\mathrm{HH}}=5.8 \mathrm{~Hz}\right), 2.64$ $\left(\mathrm{dd}, \mathrm{CHCH}_{2},{ }^{2} \mathrm{~J}_{\mathrm{HH}}=15.9,{ }^{3} \mathrm{~J}_{\mathrm{HH}}=6.6 \mathrm{~Hz}\right), 2.44\left(\mathrm{t}, 2 \mathrm{H}, \mathrm{NCH}_{2} \mathrm{CH}_{2},{ }^{3} \mathrm{~J}_{\mathrm{HH}}=7.2 \mathrm{~Hz}\right), 2.26\left(\mathrm{~s}, 3 \mathrm{H}, \mathrm{CH}_{3}\right) ;{ }^{13} \mathrm{C}$ NMR (CDCl3, $75 \mathrm{MHz})(\delta, \mathrm{ppm}) 173.1$ (C), 172.6 (C), 172.5 (C), 142.3 (C), 138.3 (C), 134.1 (C), 128.6 (CH), $123.0(\mathrm{CH}), 122.4(\mathrm{CH}), 70.9(\mathrm{CH}), 65.8(\mathrm{CH}), 52.2\left(\mathrm{CH}_{3}\right), 51.6\left(\mathrm{CH}_{3}\right), 51.6\left(\mathrm{CH}_{3}\right), 49.6$ $\left(\mathrm{CH}_{2}\right), 41.9\left(\mathrm{CH}_{2}\right), 33.4\left(\mathrm{CH}_{2}\right), 21.4\left(\mathrm{CH}_{3}\right)$. HRMS (ESI-TOF) calc. for $\mathrm{C}_{18} \mathrm{H}_{23} \mathrm{NNaO}_{6}$ requires 372.1418 , found 372.1422 .

2.7. Methyl 3-((2-methoxy-2-oxo-1-phenylethyl)amino)propanoate (6): ${ }^{1} \mathrm{H} \mathrm{NMR}\left(\mathrm{CDCl}_{3}, 300 \mathrm{MHz}\right)$ ( $\delta$, ppm) $7.24\left(\mathrm{~m}, 5 \mathrm{H}, \mathrm{C}_{6} \mathrm{H}_{5}\right), 4.31(\mathrm{~s}, 1 \mathrm{H}, \mathrm{CH}), 3.60\left(\mathrm{~s}, 3 \mathrm{H}, \mathrm{OCH}_{3}\right), 3.58\left(\mathrm{~s}, 3 \mathrm{H}, \mathrm{OCH}_{3}\right), 2.75(\mathrm{~m}, 2 \mathrm{H}$, $\left.\mathrm{NCH}_{2} \mathrm{CH}_{2}\right), 2.45$ (t, 2H, $\left.\mathrm{CH}_{2}, \mathrm{NCH}_{2} \mathrm{CH}_{2},{ }^{3} \mathrm{~J}_{\mathrm{HH}}=6.5 \mathrm{~Hz}\right) ;{ }^{13} \mathrm{C} \mathrm{NMR}\left(\mathrm{CDCl}_{3}, 75 \mathrm{MHz}\right)(\delta, \mathrm{ppm}) 172.9$ (C), $172.8(\mathrm{C}), 137.5(\mathrm{C}), 128.8(\mathrm{CH}), 128.3(\mathrm{CH}), 127.5(\mathrm{CH}), 65.4(\mathrm{CH}), 52.3\left(\mathrm{CH}_{3}\right), 51.7\left(\mathrm{CH}_{3}\right)$, $42.7\left(\mathrm{CH}_{2}\right), 34.4\left(\mathrm{CH}_{2}\right)$; HRMS (ESI-TOF) calc. for $\left[\mathrm{C}_{13} \mathrm{H}_{18} \mathrm{NO}_{4}\right]^{+}$requires 252.1230, found 252.1221.

\subsection{X-Ray crystallography. Crystal structure determination of $3 \boldsymbol{a b} \cdot \mathbf{O M}$}

Crystals of compound 3ab.OM of adequate quality for X-ray measurements were grown from diffusion of a $\mathrm{CH}_{2} \mathrm{Cl}_{2}$ solution of the crude compound in $n$-hexane at $8{ }^{\circ} \mathrm{C}$. A single crystal was mounted at the end of a quartz fiber in a random orientation, covered with perfluorinated oil and placed under a cold stream of $\mathrm{N}_{2}$ gas. The X-ray data collection was performed on an Oxford Diffraction Xcalibur2 diffractometer using graphite-monocromated Mo-K $\alpha$ radiation $(\lambda \square=0.71073$ $\AA$ ). A hemisphere of data was collected based on $\omega$-scan or $\phi$-scan runs. The diffraction frames were integrated using the program CrysAlis RED [13] and the integrated intensities were corrected for absorption with SADABS [14]. The structures were solved and developed by Patterson and Fourier methods [15]. All non-hydrogen atoms were refined with anisotropic displacement parameters. The hydrogen atoms were placed at idealized positions and treated as riding atoms. Each $\mathrm{H}$ atom was 
Revised manuscript. Article published in Journal of Molecular Catalysis A: Chemical 2017, 426, 407-418. (http://dx.doi.org/10.1016/j.molcata.2016.06.026)

assigned an isotropic displacement parameter equal to 1.2 times the equivalent isotropic displacement parameter of its parent atom. The structures were refined to $\mathrm{F}_{\mathrm{o}}{ }^{2}$ and all reflections were used in the least-squares calculations [16]. Crystallographic data (including structure factors) for the structure of 3ab.OM had been deposited with the Cambridge Crystallographic Data Centre as supplementary publication number CCDC 1474382. Copies of the data can be obtained, free of charge, on application to CCDC, 12 Union Road, Cambridge CB2 1EZ, UK (fax: +44-(0)1223-336033 or e-mail: deposit@ccdc.cam.ac.uk).

\section{Results and Discussion}

\subsection{Ru-catalyzed synthesis of isoquinoline-1-carboxylates.}

The coupling between methyl phenylglycinate (1a) and 3-hexyne (2a), catalyzed by the Ru(II) dimer $\left[\mathrm{Ru}(\mu-\mathrm{Cl}) \mathrm{Cl}\left(\eta^{6}-p \text {-cymene }\right)\right]_{2}$, [Ru], to give methyl 3,4-diethylisoquinoline-1-carboxylate (3aa) was used as model reaction for the optimization process. The starting point was the conditions previously reported by us for the Ru-catalyzed synthesis of isoquinolines from primary amines: molar ratio 1a/2a $=1 / 2,[\mathrm{Ru}](10 \%), \mathrm{KPF}_{6}(10 \%), \mathrm{Cu}(\mathrm{OAc})_{2}$ (1 equiv.), $\mathrm{MeOH}, 100{ }^{\circ} \mathrm{C}, 24 \mathrm{~h}$ [8]. Under these conditions the isoquinoline is not formed (Table 1, entry 1). Instead, a very complex mixture of decomposition products is obtained. The same result is observed when tert-amyl alcohol $\left({ }^{t} \mathrm{AmOH}\right)$ is used as solvent (entry 2). The reaction is cleaner in HFIP (hexafluoroisopropanol), affording a low 10\% yield of isolated 3aa (entry 3). This solvent has provided very good results in the coupling of alkynes with other substrates, as we have recently shown [17]. Other solvents were unable to provide higher yields.

Table 1. Optimization of the reaction conditions for the catalytic synthesis of 3aa. ${ }^{(a)}$

\begin{tabular}{|c|c|c|c|c|c|c|}
\hline Entry & Solvent & Oxidant & Temperature & time & Additives & Yield (\%) \\
\hline 1 & $\mathrm{MeOH}$ & $\mathrm{Cu}(\mathrm{OAc})_{2}$ & 100 & 24 & $\mathrm{KPF} 6$ & - \\
\hline 2 & ${ }^{t} \mathrm{AmOH}$ & $\mathrm{Cu}(\mathrm{OAc})_{2}$ & 100 & 24 & $\mathrm{KPF}_{6}$ & - \\
\hline 3 & $\mathrm{HFIP}$ & $\mathrm{Cu}(\mathrm{OAc})_{2}$ & 100 & 24 & $\mathrm{KPF}_{6}$ & 10 \\
\hline 4 & HFIP & {$\left[\mathrm{F}^{+}\right]$} & 100 & 24 & $\mathrm{KPF}_{6} / \mathrm{NaOAc}$ & 10 \\
\hline 5 & HFIP & {$\left[\mathrm{F}^{+}\right]$} & 80 & 24 & $\mathrm{KPF}_{6} / \mathrm{NaOAc}$ & 32 \\
\hline 6 & HFIP & $2 \mathrm{Ag}(\mathrm{OAc})$ & 80 & 24 & $\mathrm{KPF} 6$ & 36 \\
\hline 7 & HFIP & $2 \mathrm{AgPF} 6$ & 80 & 24 & $\mathrm{NaOAc}$ & 27 \\
\hline 8 & HFIP & $\mathrm{Ag} \mathrm{COO}_{3}$ & 80 & 24 & $\mathrm{KPF}_{6} / \mathrm{NaOAc}$ & - \\
\hline 9 & HFIP & $\mathrm{PhI}_{6}(\mathrm{OAc})_{2}$ & 80 & 24 & $\mathrm{KPF}_{6} / \mathrm{NaOAc}$ & - \\
\hline 10 & HFIP & Oxone & 80 & 24 & $\mathrm{KPF}_{6} / \mathrm{NaOAc}$ & 6 \\
\hline
\end{tabular}


Revised manuscript. Article published in Journal of Molecular Catalysis A: Chemical 2017, 426, 407-418. (http://dx.doi.org/10.1016/j.molcata.2016.06.026)

\begin{tabular}{|c|c|c|c|c|c|c|}
\hline 11 & HFIP & $2 \mathrm{Ag}(\mathrm{OAc})$ & 100 & 24 & $\mathrm{KPF}_{6}$ & 48 \\
\hline 12 & HFIP & $2 \mathrm{Ag}(\mathrm{OAc})$ & 120 & 24 & $\mathrm{KPF} 6$ & 10 \\
\hline $\mathbf{1 3}$ & HFIP & {$\left[\mathbf{F}^{+}\right]$} & $\mathbf{6 0}$ & $\mathbf{2 4}$ & $\mathbf{K P F}_{6} / \mathbf{N a O A c}$ & $\mathbf{7 3}$ \\
\hline 14 & HFIP & {$\left[\mathrm{F}^{+}\right]$} & 40 & 24 & $\mathrm{KPF}_{6} / \mathrm{NaOAc}$ & 28.5 \\
\hline 15 & HFIP & {$\left[\mathrm{F}^{+}\right]$} & 20 & 24 & $\mathrm{KPF} / \mathrm{NaOAc}$ & - \\
\hline
\end{tabular}

(a) phenylglycine methyl ester derivative 1a $(1 \mathrm{mmol})$, alkyne $2 \mathbf{a}(2 \mathrm{mmol}),[\mathrm{Ru}](10 \% \mathrm{~mol})$ in all cases; HFIP = hexafluoroisopropanol; $\left[\mathrm{F}^{+}\right]=2,4,6$-trimethylpyridinium triflate

Next we focused our attention in the correct choice of the oxidant. Our previous experience with amino acids showed that the $N$-fluoro-2,4,6-trimethylpyridinium triflate salt $\left(\left[\mathrm{F}^{+}\right]\right)$is very efficient in oxidative couplings with this type of substrates [4i]. This oxidant, however, requires a source of acetates in order to accomplish the $\mathrm{C}-\mathrm{H}$ bond activation step $[7 \mathrm{~b}, 18]$. Using $\left[\mathrm{F}^{+}\right]$in combination with $\mathrm{NaOAc}$, a similar low $10 \%$ yield is obtained (entry 4). Interestingly, a slight decrease in the reaction temperature to $80^{\circ} \mathrm{C}$ promotes a significant increase of the yield, up to $32 \%$ (entry 5), showing that decomposition pathways are probably competing with the annulation process. Other oxidants were tested. Silver salts $\mathrm{AgOAc}$ and $\mathrm{AgPF}_{6}$ in combination with $\mathrm{NaOAc}$ (entries 6 and 7) give 3aa in isolated yields comparable to those obtained with the fluoropyridinium salt. However, $\mathrm{Ag}_{2} \mathrm{CO}_{3}$ (entry 8 ) and other classical oxidants such as $\mathrm{PhI}(\mathrm{OAc})_{2}$ (entry 9) or oxone (entry 10) were ineffective.

Therefore, both AgOAc and $\left[\mathrm{F}^{+}\right]$(in combination with $\mathrm{NaOAc}$ ), show the same efficiency but still need additional improvement. The isolated yield of 3aa increases up to 48\% (entry 11) when AgOAc is used as oxidant by increasing the temperature to $100{ }^{\circ} \mathrm{C}$. Further increasing, however, does not have a positive effect (entry 12 ). In clear contrast, the effect of temperature in the reaction with $\left[\mathrm{F}^{+}\right]$ is the opposite. Expectedly, by decreasing the temperature to $60{ }^{\circ} \mathrm{C}$ the yield increases (up to $73 \%$, entry 13), probably because the competing decomposition pathways are minimized. Longer reaction times (48 h) afford virtually the same yield. However, a further decrease of the reaction temperature gave poorer conversions and, consequently, lower isolated yields (entries 14 and 15). At $40{ }^{\circ} \mathrm{C}$ the isolated yield is only around $28 \%$, while at room temperature the reaction does not take place and starting $\mathbf{1 a}$ is recovered.

Once the optimal reaction conditions for the oxidative coupling of methyl phenylglycinate (1a) and 3-hexyne (2a) to give the isoquinoline-1-carboxylate (3aa) have been determined, the scope of the reaction has been examined. As for the alkynes, the reaction works with electron-rich alkynes and aromatic alkynes. The results are summarized in Figure 2. Diphenylacetylene (2b) reacts with $\mathbf{1 a}$ to give 3,4,-diphenylisoquinolinone-1-carboxylate (3ab) in a modest $40 \%$ yield, and a similar yield of 3(4)-methyl-4(3)-propylisoquinoline-1-carboxylate (3ac) was obtained from the coupling of 2- 
hexyne (2c) with 1a (42\%). Gratifyingly, better yields were obtained for coupling involving 1phenylpropyne (2d), giving the corresponding isoquinoline 3ad in 64\% yield. In the same way, 1a reacted with 4,4-dimethyl-2-pentyne (2e), giving three different coupling products $\mathbf{3 a e}$ in a global $80 \%$ yied. Only the monoinsertion of alkynes in type $\mathbf{3}$ products has been observed in all studied cases. This selectivity is worthy of note because in analogous reactions with benzylamines a double alkyne insertion took place, affording 8-vinyl-isoquinolines [8]. Regarding asymmetric alkynes, the regioselectivity of the insertion depends on the alkyne. Approximately equimolar amounts of the two regioisomers are obtained when 2-hexyne is employed (3ac). On the other hand, a remarkable selectivity was observed in the case of 1-phenylpropyne (3ad), the most abundant isomer being that with the phenyl ring close to the $\mathrm{N}$ atom, probably due to steric factors and in keeping with previous observations in closely related systems [8a].

The reaction of $\mathbf{1 a}$ with 4,4-dimethyl-2-pentyne (2e) deserves some additional comments. The crude reaction mixture contains three different species (Figure 2), which are easily separated by column chromatography. Two of them are the expected regioisomers of methyl (tert-butyl)methylisoquinoline-1-carboxylate (3ae1 and 3ae2), obtained in 15\% and 25\% yield, respectively, while NMR characterization of the third compound revealed that it is methyl 1-amino-2-(tert-butyl)3-methyl-1H-indene-1-carboxylate (3ae3), obtained in 40\% isolated yield. Therefore the combined yield of this reaction is about $80 \%$. 
Revised manuscript. Article published in Journal of Molecular Catalysis A: Chemical 2017, 426, 407-418. (http://dx.doi.org/10.1016/j.molcata.2016.06.026)

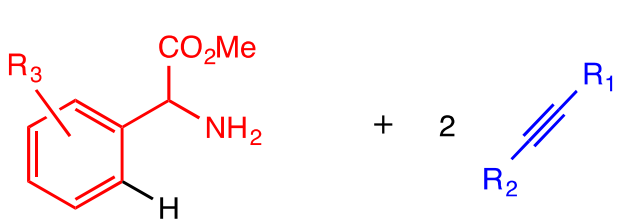

$\mathrm{R}_{3}=\mathrm{H}$ 1a, 3-NO 1 1b, 4-Cl 1c, $\mathrm{R}_{1}=\mathrm{R}_{2}=\mathrm{Et} \mathrm{2a}, \mathrm{Ph} \mathbf{2 b}$

3-Br 1d, 4-OMe 1e, 4-Me 1f $\quad R_{1}=M e ; R_{2}={ }^{n} \operatorname{Pr} 2 c$, Ph 2d, tBu $2 e$

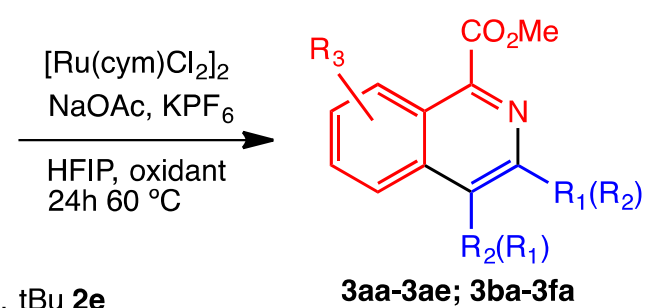

3aa-3ae; 3ba-3fa<smiles>CCc1nc(C(C)=O)c2ccccc2c1CC</smiles>

3aa $(73 \%)$<smiles>CC(=O)c1nc(-c2ccccc2)c(-c2ccccc2)c2ccccc12</smiles>

$3 \mathrm{ab}(40 \%)$<smiles>CC(=O)c1nc(C)c(C(C)C)c2ccccc12</smiles>

3ac $(42 \%)$

(3ac1 3-Me: 17\%; 3ac2 4-Me: 25\%)<smiles>CC(=O)c1nc(-c2ccccc2)c(C)c2ccccc12</smiles>

(3ad1 3-Me: 13\%; 3ad2 4-Me: 51\%)<smiles>CC(=O)c1nc(C(C)=O)c2ccccc2c1C(=O)OC(C)(C)C</smiles>

(3ae1 3-Me: 15\%; 3ae2 4-Me: 25\%)<smiles>CC(=O)c1nc(C(C)C)c(C)c2ccccc12</smiles><smiles>CCc1nc(C(C)=O)c2cc([N+](=O)[O-])ccc2c1CC</smiles>

3ba (41\%)<smiles>CCc1nc(C(C)=O)c2ccc(Cl)cc2c1CC</smiles>

3ca $(60 \%)$<smiles>CCc1nc(C(C)=O)c2cc(Br)ccc2c1CC</smiles><smiles>CCc1nc(C(C)=O)c2cccc(Br)c2c1CC</smiles>

3 da $(33 \%)$

(3da1 7-Br: 25\%; 3da2 5-Br: 8\%)<smiles>CCc1nc(C(C)=O)c2ccc(OC)cc2c1CC</smiles>

3ea $(72 \%)$<smiles>CCc1nc(C(C)=O)c2ccc(C)cc2c1CC</smiles>

3 fa $(75 \%)$

Figure 2. Scope of the oxidative coupling to give isoquinoline-1-carboxylates 3

The regiochemistry of $\mathbf{3 a e} \mathbf{1}$ and $\mathbf{3 a e} \mathbf{2}$ has been determined by selective 1D-NOESY measurements (see Supporting Information) and shows that the most abundant isomer is that with the ${ }^{\mathrm{t}} \mathrm{Bu}$ group adjacent to the nitrogen atom. This is consistent with previous results obtained in our group, since this orientation is the same than that observed in related Ru-catalyzed synthesis of N-heterocycles [8], and in reported stoichiometric Pd insertions [19a]. However, the regiochemistry here observed is the 
opposite to that determined in Ru-mediated stoichiometric synthesis of isoquinolinium salts [19b]. The subtle differences in the Ru-complexes $\left[\left(\eta^{6}\right.\right.$-benzene $) \mathrm{Ru} v s\left(\eta^{6}\right.$-cymene $\left.) \mathrm{Ru}\right]$, as well as in the substrates ( $N, N$-dimethylbenzylamines $v s$ phenylglycine), could account for the different reactivity, as it has been shown that the regioselectivity in the insertion of this alkyne is largely dependent on steric factors.

The formation of different compounds 3ae1-3ae3 involves two different reaction mechanisms which share the initial steps of the catalytic cycle: the Ru-promoted and acetate-assisted ortho- $\mathrm{CH}$ bond activation of the methyl phenylglycinate [18] and the migratory insertion of the internal alkyne into the Ru-C bond (Figure 3). This results in the formation of a cycloruthenated intermediate. From this species the reductive elimination can take place according to our previous mechanistic determinations [8]. The C-N coupling involves the decoordination of the $\mathrm{N}$ atom and subsequent nucleophilic attack to the metallated vinylic carbon, giving a dihydroisoquinolonium salt and a $R u(0)$ fragment which is then oxidized to $\mathrm{Ru}(\mathrm{II})$ by the pyridinium salt. Next, the dihydroisoquinolonium salt further evolves by deprotonation to give a dehydroisoquinoline, which aromatizes by dehydrogenation affording the isoquinolines 3ae1 and 3ae2. On the other hand, the formation of 3ae3 strongly suggests an alternative reaction pathway, also depicted in Figure 3. This alternative mechanism involves the deprotonation of the $\mathrm{C} \alpha$ of the orthoruthenated phenylglycine and further attack of the carbanion to a vinylic carbon giving the indene unit. The formation of the carbanion is explained by the presence of an excess of base (acetate) and by the acidic nature of the $\mathrm{H}$ on the alpha carbon. After the C-C coupling the corresponding $\mathrm{Ru}(0)$ species is similarly oxidized to $\mathrm{Ru}(\mathrm{II})$ by the pyridinium salt. Although the formation of the indene derivative is observed in the largest extent in this case (3ae3), the presence of analogous indene side-products in other cases cannot be totally discarded. 


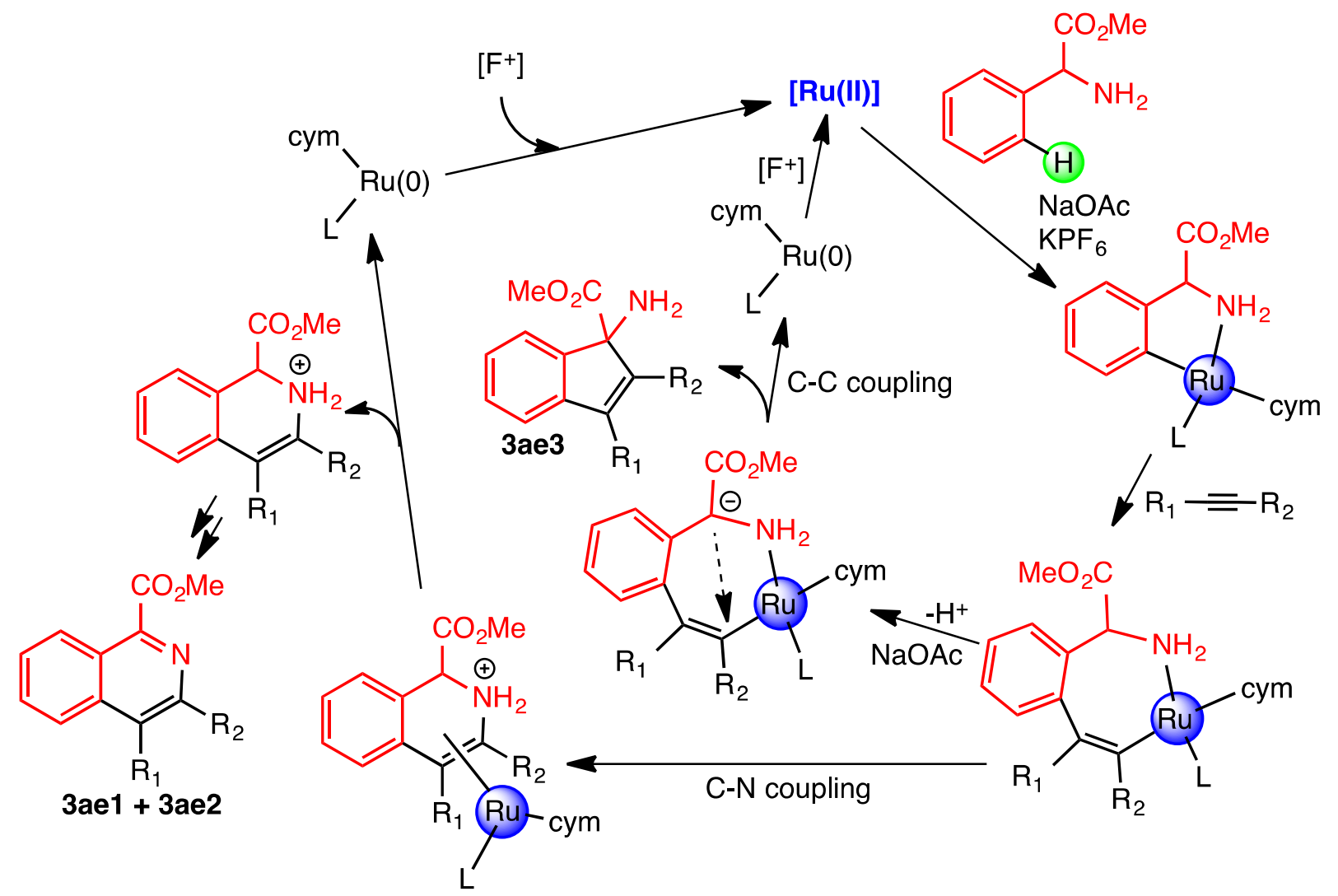

Figure 3. Proposed mechanistic pathways to explain the formation of isoquinoline and indene derivatives

As for the scope of phenylglycines, the reaction works for substrates which contain both electronattracting and electron-releasing substituents. Good yields are obtained when methoxy and methyl substituents are present in para position of the starting materials (3ea $72 \%$ and 3 fa $75 \%$ respectively). Slightly lower isolated yields are obtained when the chloride group is in para position (3ca, 60\%). In this case the likely product of dechlorination (3aa) was not observed, showing that the reaction has a notable tolerance for different functional groups. Moreover, the presence of the chlorine atom allows further orthogonal reactivity, for instance through Heck or Suzuki processes. On the other hand, only moderate yields were achieved when bromide or the strong electron-attracting nitro group are in meta positions (3da 33\% and 3ba 41\%). Interestingly, the reaction of the nitro derivative is regioselective, and only the less hindered product $\mathbf{3 b a}$ with the nitro group at 7-position is obtained, while in the case of the phenylglycinate containing a bromine atom, the two possible regioisomers (incorporating bromine in 5- and in 7-positions), were obtained. As expected, the less hindered 7-Br isomer was formed in higher amount (3.1/1 molar ratio). 
Revised manuscript. Article published in Journal of Molecular Catalysis A: Chemical 2017, 426, 407-418. (http://dx.doi.org/10.1016/j.molcata.2016.06.026)

\subsection{Ru-catalyzed synthesis of isoindoline-1-carboxylates (5aa-5fa).}

Prompted by the remarkable results obtained in the synthesis of isoquinoline-1-carboxylates 3 by oxidative coupling, we have attempted the analogous insertion reactions using alkenes. The most important results obtained in this reaction are presented in Figure 4.

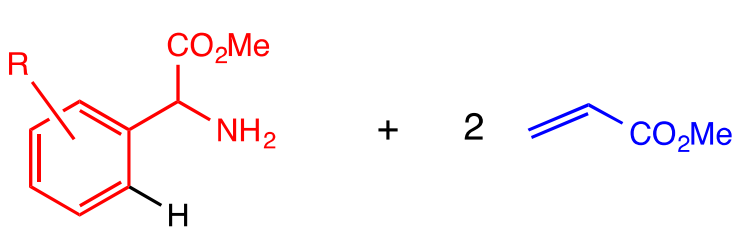

$\mathrm{R}_{3}=\mathrm{H} 1 \mathrm{a}, 3-\mathrm{NO}_{2} 1 \mathrm{~b}, 4-\mathrm{Cl} 1 \mathrm{c}$,

3-Br 1d, 4-OMe 1e, 4-Me 1f

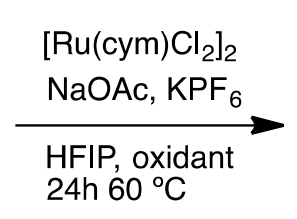

$4 a$

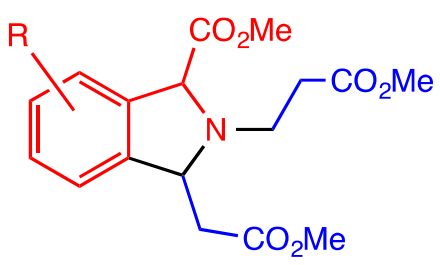

5aa-5fa<smiles>COC(=O)c1ccccc1</smiles>

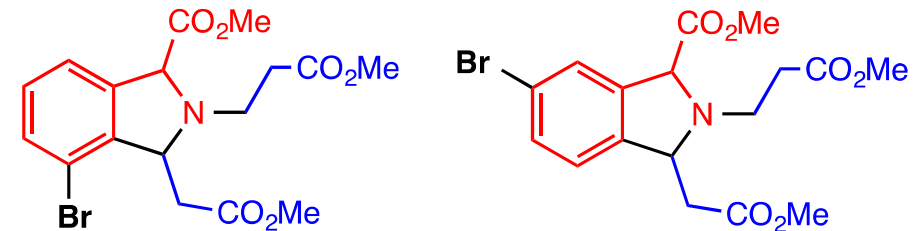

5da (38\%) (4-Br: 12\%; 6-Br: $26 \%)$ d.r. for the major isomer $=38 \%$<smiles>CC(=O)CCN1C(CC(C)=O)c2cc(C)ccc2C1C(C)=O</smiles>

5 fa $(66 \%)$ d.r. $=80 \%$

Figure 4. Scope of the oxidative coupling to give isoindoline-1-carboxylates 5

Using the same reaction conditions than those described for the synthesis of isoquinolines $\mathbf{3}$, phenylglycines 1a-1f react with methyl acrylate 4a (1:2 molar ratio) to give the corresponding isoquinoline-1-carboxylates 5aa-5fa in yields up to 66\% (Figure 4). Since some of the yields are quite low, a further optimization of the reaction parameters was attempted, but the effort proved ineffective. Nevertheless, in all the studies cases the reaction takes place with full conversion of the starting amino ester 1, and NMR yields calculated from the reaction crude are higher than the isolated yields reported in Figure 4. The difficulties which arose during the purification step (by column chromatography) and the formation of side-products (as detailed below) account for this disagreement.

The scope of the studied phenylglycines is depicted in Figure 4. Substituents of electron-withdrawing and electron-donating nature are tolerated, although better yields are obtained for methyl (5fa) and methoxy (5ea) groups (66\% and 44\% yield, respectively) than for chloride (5ca), bromide (5da) or 
nitro (5ba) moieties (37\%, 38\% and 27\%). Regarding the alkenes, the scope is limited to acrylates. Alkenes bearing other strong electron-attracting functional groups (phosphonate, sulfonate, sulfone) or relatively more electron-rich alkenes (for instance, styrene) did not show significant reactivity. In all the cases the reaction takes place with the incorporation of two equivalents of alkene $4 \mathbf{a}$. One of the alkenes is consumed by reaction with the amino group through a classical hydroamination or aza-Michael addition process. This step occurs without the metal mediation, as has been confirmed by the direct treatment of $1 \mathbf{a}$ with $4 \mathbf{a}$, affording the expected $N$-protected phenylglycine $\mathbf{6}$ (Figure 5). Surprisingly, the reaction of $\mathbf{6}$ with acrylate $4 \mathbf{a}$ under the optimized catalytic conditions does not afford the isoquinoline-1-carboxylate product 5aa. This fact suggests that $N$-protected phenylglycine $\mathbf{6}$ is not an intermediate in the synthesis of $\mathbf{5 a a}$, and that hydroamination takes place in a late stage of the reaction.
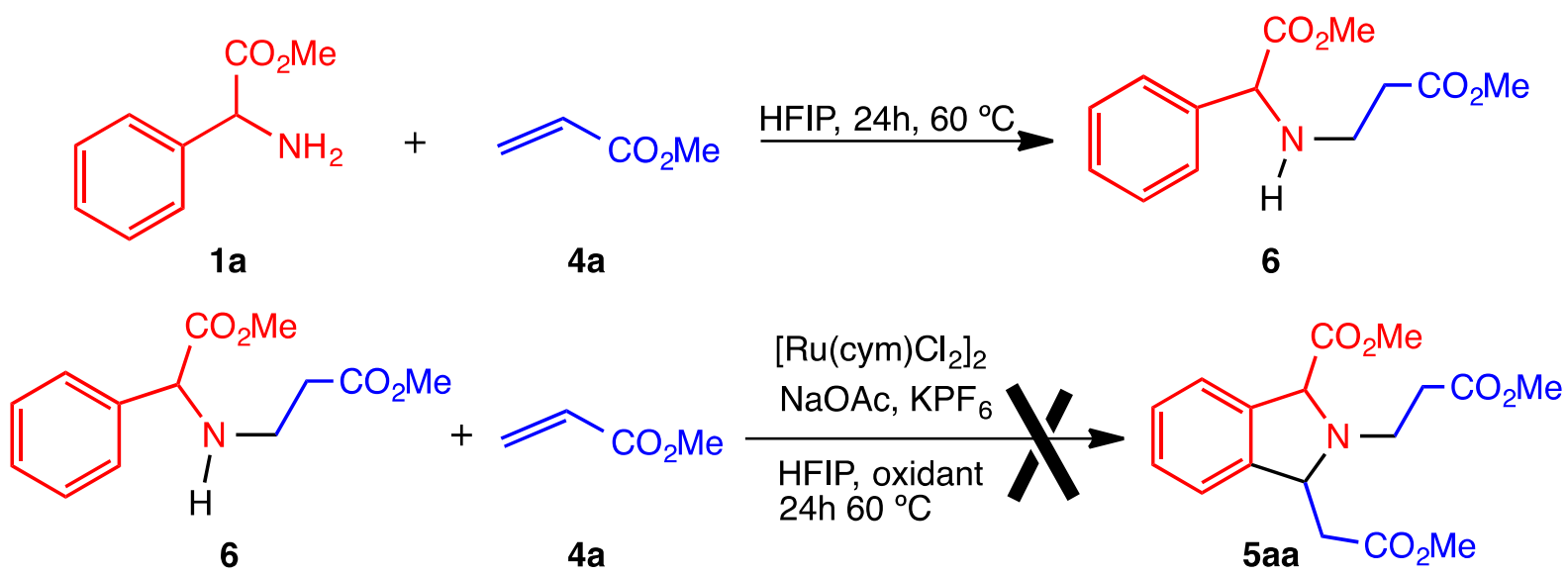

Figure 5. Some control experiments in the synthesis of isoquinoline-1-carboxylates 5

According to these observations, we propose that the first equivalent of alkene reacts with phenylglycine 1a to give the corresponding Heck intermediate A, shown in Figure 6. This reaction takes place presumably through the cycloruthenation of $\mathbf{1 a}$ by $\mathrm{C}-\mathrm{H}$ activation, followed by migratory insertion of the alkene and $\beta$-elimination from the corresponding alkyl derivative to yield $\mathbf{A}$. After that, A may undergo two different pathways, none of which can be ruled out with the present data: either the intramolecular aza-Michael addition affording isoindoline $\mathbf{B}$, followed by intermolecular amination to afford 5aa or, alternatively, the intermolecular aza-Michael coupling to form intermediate $\mathbf{C}$ which finally yields $\mathbf{5} \mathbf{a a}$ by an intramolecular hydroamination process. 


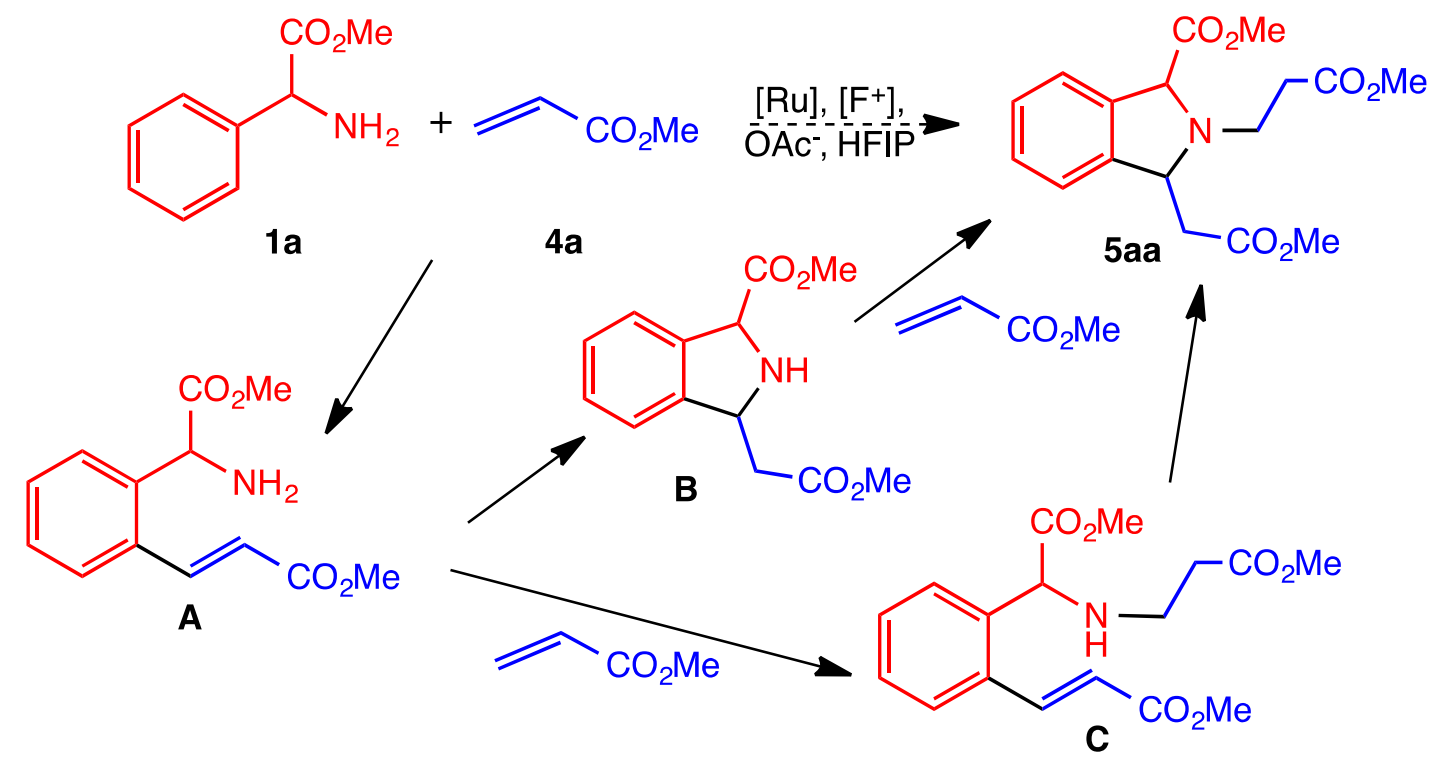

Figure 6. Proposed pathways for the oxidative synthesis of isoindoline-1-carboxylates 5

Isoindolines $\mathbf{5}$ have two stereogenic centers and are obtained as mixtures of diastereoisomers, as it is clear from their ${ }^{1} \mathrm{H}$ NMR spectra. The values of diastereomeric excess are in the range of $50-80 \%$, proving that the reaction occurs with a notable degree of diastereoselectivity. In order to get more information about the relative configurations of the new stereogenic centers, selective NOE experiments have been carried out in the major diastereoisomer of 5ca (see Supporting Information). The perturbation of the signal at $4.60 \mathrm{ppm}(\mathrm{C} 1-H)$ produces NOE enhancements in the ester groups at 3.60 and $3.67 \mathrm{ppm}$, meaning that the substituents in $\mathrm{C} 1\left(\mathrm{CO}_{2} \mathrm{Me}\right)$ and in $\mathrm{C} 3\left(\mathrm{CH}_{2} \mathrm{CO}_{2} \mathrm{Me}\right)$ are in mutual trans positions. Therefore, the relative configurations in the major diastereoisomer are $(1 S, 3 R / 1 R, 3 S)$. This result is agrees with previous observations in very closely related systems, and probably accounts for a minimization of the steric interactions between the different substituents [4i].

\subsection{Characterization of the crude reaction products}

The two preceding sections show that Ru(II) complexes are efficient catalysts for the site-selective transformation of $\mathrm{N}$-unprotected amino acids, such as phenylglycine, through $\mathrm{C}-\mathrm{H}$ bond activation reactions. The successful synthesis of isoquinoline-1-carboxylates $\mathbf{3}$ and isoindoline-1-carboxylates 5 provides the proof-of-concept that opens the door for future and diverse modifications using Rucatalysts. However, the yields obtained in some cases are low, even under optimized reaction conditions, indicating the existence of additional processes competing against the target reaction. The analysis of crude mixtures, prior to purification, provides important clues on the nature of these sidereactions. Our analysis show, in many cases, the presence of variable amounts of organometallic species of $\mathrm{Ru}(\mathrm{II})$ coordinated to the $\eta^{6}$-cymene ligand, in addition to the expected organic derivatives. 
Revised manuscript. Article published in Journal of Molecular Catalysis A: Chemical 2017, 426, 407-418. (http://dx.doi.org/10.1016/j.molcata.2016.06.026)

We were able to isolate one of these organometallic derivatives from the reaction of phenylglycine 1a with diphenylacetylene $\mathbf{2 b}$. After isoquinoline $\mathbf{3 a b}$ has been separated and purified by column chromatography, further elution with a mixture $\mathrm{CH}_{2} \mathrm{Cl}_{2} / \mathrm{MeOH}$ (see Experimental) allows to isolate a brown fraction. Deep red crystals of 3ab.OM were obtained from crystallization of the brown fraction in $\mathrm{CH}_{2} \mathrm{Cl}_{2}$ /hexane, and were of enough quality for the determination of the crystal structure by X-ray diffraction methods. The drawing of the organometallic complex 3ab.OM is shown in Figure 8, with selected bond distances and angles.

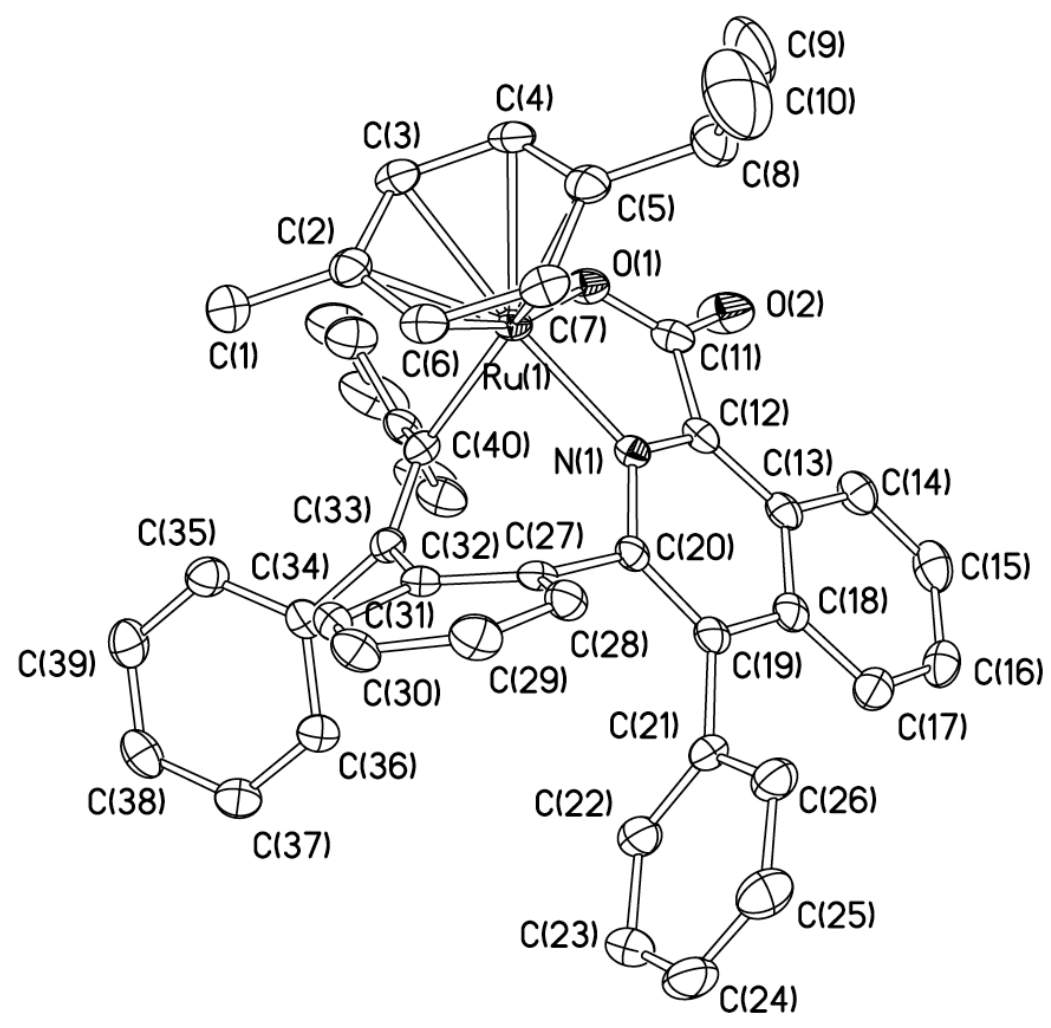

Figure 8. Drawing of organometallic compound 3ab.OM. Selected bond distances $[\AA]$ and angles [']: C2-Ru1 2.239(3), C3-Ru1 2.202(3), C4-Ru1 2.230(3), C5-Ru1 2.302(3), C6-Ru1 2.209(3), C7Ru1 2.159(3), C40-Ru1 2.085(3), N1-Ru1 2.084(2), O1-Ru1 2.067(2), O1-Ru1-N1 76.68(7), O1Ru1-C40 90.15(9), N1-Ru1-C40 78.20(9).

The structure shows that the $\mathrm{Ru}(\mathrm{II})$ center is located in a three-legged piano-stool environment (basically a distorted octahedral environment) in which one $\mathrm{Ru}-\mathrm{C}$ bond, one $\mathrm{Ru}-\mathrm{N}$ bond and one $\mathrm{Ru}-$ $\mathrm{O}$ bond make up the three legs and the $\eta^{6}$-cymene ligand the seat of the stool. The cymene ligand shows the expected $\eta^{6}$-bonding mode and the internal bond distances and angles are similar to those found in closely related examples [8b,20]. However, the C,N,O-tridentate ligand that occupies the other three coordination sites is remarkable. It is likely that this ligand has been formed in several consecutive steps. The first one may consist in the cycloruthenation of phenylglycine 1a by ortho-C- 
$\mathrm{H}$ bond activation, migratory insertion of diphenylacetylene and reductive elimination of 3,4diphenyl-isoquinoline-1-carboxylate fragment (3ab). This isoquinoline is then able to undergo a second $\mathrm{C}-\mathrm{H}$ activation at the ortho position of the 3-phenyl ring and a subsequent migratory insertion of a second equivalent of diphenylacetylene, forming a vinyl seven-membered ring. Demethylation of the ester function by saponification can take place at any stage of this sequence; the reactions are performed in the presence of base (NaOAc) in a protic and acidic solvent (HFIP), and the presence of water in the solvent cannot be excluded.

Although we have not isolated any final products arising from a double alkyne insertion, complex 3ab.OM proves that they are actually formed. This fact could explain the low yields observed in some of the examples, because reagents (alkyne) are being consumed by forming these side-products, the target product (3ab) is being removed from the reaction medium and $\mathrm{Ru}$ is being sequestered in the form of stable complexes, thus decreasing the effective concentration of the catalyst. Further work with the aim of decreasing the extent of this type of side-reactions is currently being developed in our laboratory.

\section{Conclusions}

The $\mathrm{C}-\mathrm{H}$ functionalization of $\mathrm{N}$-unprotected amino acids, such as methyl phenylglycinate, is achievable using ruthenium complexes as catalysts. Two different processes have been optimized. The Ru-catalyzed oxidative coupling of methyl arylglycinates $\mathbf{1}$ with internal alkynes $\mathbf{2}$ affords the corresponding substituted isoquinoline-1-carboxylates 3, for a wide scope of alkynes and arylsubstituted methyl arylglycinates. The selective incorporation of only one unit of alkyne takes place even though, in the case of asymmetric alkynes, the regioselectivity of the insertion is modest. On the other hand the Ru-catalyzed oxidative coupling of methyl arylglycinates $\mathbf{1}$ with electron-poor alkenes 4 gives rise to the substituted isoindoline-1-carboxylates 5, for different aryl-substituted methyl arylglycinates.

\section{Acknowledgments}

Funding by the Ministerio de Economia y Competitividad (MINECO) (Spain, Project CTQ201340855-R) and Gobierno de Aragón-Fondo Social Europeo (Spain, groups E40 and E97) is gratefully acknowledged. S. R. thanks Gobierno de Aragón for a PhD grant. Authors would also like to acknowledge the use of Servicio General de Apoyo a la Investigación-SAI, Universidad de Zaragoza. 
Revised manuscript. Article published in Journal of Molecular Catalysis A: Chemical 2017, 426, 407-418. (http://dx.doi.org/10.1016/j.molcata.2016.06.026)

\section{References}

[1] a) Amino Acids, Peptides and Proteins in Organic Chemistry, A.B. Hughes, Editor; WileyVCH: Weinheim, 2009-2013; Vols. 1-5; b) Handbook on Synthesis of Amino Acids: General Routes for the Syntheses of Amino Acids, M. Blaskovich; Oxford University Press: New York, 2010; c) Asymmetric Synthesis and Application of $\alpha$-Amino Acids, V.A. Soloshonok, K. Izawa, Editors; American Chemical Society: Washington DC, 2009; Vol.1009.

[2] C. Nájera, J.M. Sansano, Chem. Rev. 107 (2007) 4584.

[3] For a specific review: a) A.F.M. Noisier, M.A. Brimble, Chem. Rev. 114 (2014) 8775. For general reviews about the relevance of $\mathrm{C}-\mathrm{H}$ bond activation in organic synthesis see: b) $\mathrm{C}-\mathrm{H}$ Activation, J.-Q. Yu, Z. Shi, Editors, Top. Curr. Chem. 292 (2010) 1-379; c) J. Wencel-Delord, F. Glorius, Nature Chem. 5 (2013) 369; d) C-H Bond Activation and Catalytic Functionalization I, P.H. Dixneuf, H. Doucet, Editors, Top. Organomet. Chem. 55 (2016) 1-260; e) C-H Bond Activation and Catalytic Functionalization II, P.H. Dixneuf, H. Doucet, Editors, Top. Organomet. Chem. 56 (2016) 1-207; f) J.F. Hartwig, J. Am. Chem. Soc. 138 (2016) 2; f) C-H Bond Activation in Organic Synthesis, J.J. Li, Editor; CRC Press, Taylon and Francis Group, Boca Ratón, 2015.

[4] For catalytic $\mathrm{C}\left(\mathrm{sp}^{2}\right)-\mathrm{H}$ functionalization of aromatic $\alpha$-amino acid derivatives, see: a) J.J. Li, T.-S. Mei, J.-Q. Yu, Angew. Chem., Int. Ed. 47 (2008) 6452 (iodination, amination, alkenylation); b) T.-S. Mei, X. Wang, J.-Q. Yu, J. Am. Chem. Soc. 131 (2009) 10806 (amination); c) F.-M. Meyer, S. Liras, A. Guzmán-Perez, C. Perreault, J. Bian, K. James, Org. Lett. 12 (2010) 3870 (borylation); d) C. J. Vickers, T.-S. Mei, J.-Q. Yu, Org. Lett. 12 (2010) 2511 (acetoxylation, amination); e) B. López, A. Rodriguez, D. Santos, J. Albert, X. Ariza, J. García, J. Granell, Chem. Commun. 47 (2011) 1054 (carbonylation); f) G. He, Y. Zhao, S. Zhang, C. Lu, G. Chen, J. Am. Chem. Soc. 134 (2012) 3 (amination); g) J. Albert, X. Ariza, T. Calvet, M. Font-Bardia, J. García, J. Granell, A. Lamela, B. López, M. Martinez, L. Ortega, A. Rodriguez, D. Santos, Organometallics 32 (2013) 649 (carbonylation); h) T.-S. Mei, D. Leow, H. Xiao, B.N. Laforteza, J.-Q. Yu, Org. Lett. 15 (2013) 3058 (amination); i) A. García-Rubia, E. Laga, C. Cativiela, E.P. Urriolabeitia, R. Gómez-Arrayás, J.C. Carretero, J. Org. Chem. 80 (2015) 3321 (alkenylation); j) N. Dastbaravardeh, T. Toba, M.E. Framer, J.-Q. Yu, J. Am. Chem. Soc. 137 (2015) 9877; k) K.-J. Xiao, L. Chu, J.-Q. Yu, Angew. Chem. Int. Ed. 55 (2016) 2856.

[5] Price of Pd (2016 May 17) was 520.05 €/ozt (www.infomine.com/investment/metalprices/palladium/), while prices of $\mathrm{Rh}$, Ir and $\mathrm{Ru}$ at the same moment were $595.76 € / \mathrm{ozt}, 458.96 € / \mathrm{ozt}$ and $37.07 € /$ ozt, respectively.

[6] E. Laga, A. García-Montero, F.J. Sayago, T. Soler, S. Moncho, C. Cativiela, M. Martínez, E.P. Urriolabeitia, Chem. Eur. J. 19 (2013) 17398. 
Revised manuscript. Article published in Journal of Molecular Catalysis A: Chemical 2017, 426, 407-418. (http://dx.doi.org/10.1016/j.molcata.2016.06.026)

[7] State-of-the-art in ruthenium, recent reviews: a) Ruthenium in Catalysis, C. Bruneau, P.H. Dixneuf Editors, Top. Organomet. Chem. 48 (2014) 1-397; b) L. Ackermann, Chem. Rev. 111 (2011) 1315; c) P.B. Arockiam, C. Bruneau, P.H. Dixneuf, Chem. Rev. 112 (2012) 5879; d) B. Li, P.H. Dixneuf, Chem. Soc. Rev. 42 (2013) 5744; e) S.I. Kozhushkov, L. Ackermann, Chem. Sci. 4 (2013) 886; f) C. Gunananthan, D. Milstein, Chem. Rev. 114 (2014) 12024; g) S. De Sarkar, W. Liu, S.I. Kozhushkov, L. Ackermann, Adv. Synth. Catal. 356 (2014) 1461; h) L. Ackermann, Acc. Chem. Res. 47 (2014) 281; i) A. Biafora, F.W. Patureau, Synlett 25 (2014) 2525; j) V.S. Thirunavukkarasu, S.I. Kozhushkov, L. Ackermann, Chem. Commun. 50 (2014) 29; k) Y. Rao, G. Shan, X.-L. Yang, Sci. China Chem. 57 (2014) 930; 1) L. Ackermann, Org. Process Res. Dev. 19 (2015) 260; m) C. Liu, J. Yuan, M. Gao, S. Tang, W. Li, R. Shi, A. Lei, Chem. Rev. 115 (2015) 12138; n) R. Manikandan, M. Jeganmohan, Org. Biomol. Chem. 13 (2015) 10420; o) A. Molnar, A. Papp, Curr. Org. Chem. 20 (2016) 381.

[8] a) P. Villuendas, E.P. Urriolabeitia, J. Org. Chem. 78 (2013) 5254; b) S. Ruiz, P. Villuendas, M.A. Ortuño, A. Lledós, E.P. Urriolabeitia, Chem. Eur. J. 21 (2015) 8626.

[9] a) M.A. Bennett, A.K. Smith, J. Chem. Soc., Dalton Trans. (1974) 233. b) A. Kudelko, W. Zieliński, K. Ejsmont, Tetrahedron 67 (2011) 7838; c) S. Nieto, F.J. Sayago, P. Laborda, T. Soler, C. Cativiela, E.P. Urriolabeitia, Tetrahedron 67 (2011) 4185.

[10] G. Dotsevi, Y. Sogah, D.J. Cram, J. Am. Chem. Soc. 98 (1976) 3038.

[11] Y. Wen, Y. Xiong, L. Chang, J. Huang, X. Liu, X. Feng, J. Org. Chem. 72 (2007) 7715.

[12] M. Penso, D. Albanese, D. Landini, V. Lupi, A. Tagliabue, J. Org. Chem. 73 (2008) 6686.

[13] CrysAlis RED, version 1.171.27p8; Oxford Diffraction Ltd., Oxford, U.K., 2005.

[14] G.M. Sheldrick, SADABS, Program for absorption and other corrections, Göttingen University, 1996.

[15] G.M. Sheldrick, SHELXS-86, Acta Crystallogr. A46 (1990) 467.

[16] G.M. Sheldrick, SHELXL-97, Acta Crystallogr. A64 (2008) 112.

[17] P. Villuendas, E.P. Urriolabeitia, Org. Lett. 17 (2015) 3178.

[18] a) Y. Boutadla, D.L. Davies, S.A. Macgregor, A.I. Poblador-Bahamonde, Dalton Trans. (2009) 5820; b) D. Lapointe, K. Fagnou, Chem. Lett. 39 (2010) 1118; c) S.I. Gorelsky, D. Lapointe, K. Fagnou, J. Org. Chem. 77 (2012) 658; d) D. García-Cuadrado, A.A.C. Braga, F. Maseras, A.M. Echavarren, J. Am. Chem. Soc. 130 (2008) 10848; e) E. Ferrer Flegeau, C. Bruneau, P.H. Dixneuf, A. Jutand, J. Am. Chem. Soc. 133 (2011) 10161; f) I. Fabre, N. von Wolff, G. Le Duc, E. Ferrer Flegeau, C. Bruneau, P.H. Dixneuf, A. Jutand, Chem Eur. J. 19 (2013) 7595. 
Revised manuscript. Article published in Journal of Molecular Catalysis A: Chemical 2017, 426, 407-418. (http://dx.doi.org/10.1016/j.molcata.2016.06.026)

[19] a) J. Spencer, M. Pfeffer, N. Kyritsakas, J. Fischer, Organometallics 14 (1995) 2214; b) H.C.L. Abbenhuis, M. Pfeffer, J.P. Sutter, A. deCian, J. Fischer, H.L. Ji, J.H. Nelson, Organometallics 12 (1993) 4464.

[20] L. Cuesta, T. Soler, E.P. Urriolabeitia, Chem. Eur. J. 18 (2012) 15178. 